\title{
GLAD!
}

Revue sur le langage, le genre, les sexualités

$10 \mid 2021$

Varia

\section{Démêler les effets des stéréotypes et le genre grammatical dans le biais masculin : une approche expérimentale}

Untangling Stereotype Effects from Masculine Grammatical Gender Bias in

French: an Experimental Approach

\section{Célia Richy et Heather Burnett}

\section{OpenEdition \\ Journals}

Édition électronique

URL : https://journals.openedition.org/glad/2839

DOI : 10.4000/glad.2839

ISSN : 2551-0819

Éditeur

Association GSL

\section{Référence électronique}

Célia Richy et Heather Burnett, « Démêler les effets des stéréotypes et le genre grammatical dans le biais masculin : une approche expérimentale », GLAD! [En ligne], 10 | 2021, mis en ligne le 15 juillet 2021, consulté le 26 juillet 2021. URL : http://journals.openedition.org/glad/2839 ; DOI : https:// doi.org/10.4000/glad.2839

Ce document a été généré automatiquement le 26 juillet 2021.

\section{(c) (†) $\odot$}

La revue GLAD! est mise à disposition selon les termes de la Licence Creative Commons Attribution Pas d'Utilisation Commerciale - Pas de Modification 4.0 International. 


\title{
Démêler les effets des stéréotypes et le genre grammatical dans le biais masculin : une approche expérimentale
}

\author{
Untangling Stereotype Effects from Masculine Grammatical Gender Bias in \\ French: an Experimental Approach
}

Célia Richy et Heather Burnett

\section{Introduction}

L'effet du genre grammatical sur les représentations mentales des locutrices et locuteurs suscite un intérêt croissant dans le domaine de la recherche francophone en linguistique et psycholinguistique (e.g. Chatard et al. 2005; Brauer et Landry 2008 ; Gygax et al. 2008 ; Gygax et al. 2012 ; Gygax et al. 2019). Selon une perspective formelle, dans son acception large, le genre grammatical permet de classer les noms en fonction $\mathrm{du}$ "behavior of associated words" (Hockett, 1958: 231), autrement dit selon le type d'accord déclenché par le nom sur ses dépendants. Martinet (1999) décrit le système de genre grammatical du français comme plutôt inutile: "l'information qu'apporte aux usagers de la langue l'existence des genres féminin et masculin est pratiquement nulle » (ibid. : 9). Il argumente que le genre grammatical apporte en réalité peu de «valeur informative " (ibid.: 7) comparé à la lourdeur du système morphologique et aux complications d'accord qu'il entraine, et ce parce que peu de mots (homophones) requièrent l'utilisation du genre grammatical pour désambigüiser leur sens (1). En outre, selon l'auteur, le sens de ces mots peut en réalité être reconstruit en fonction des contextes où ils apparaissent.

(1) a. La livre vs le livre

b. La voile vs le voile 
Cela étant, associé à des référents humains, le genre grammatical peut revêtir une fonction particulière: de l'encodage du genre social découle une variété de sens, d'implications et de connotations. Par exemple, Schafroth (2003) décrit le phénomène de functional doublets, où pour un même nom le genre grammatical délimite ce qui est prestigieux et ce qui ne l'est pas (2).

(2) La couturière vs le couturier

3 Pourtant, la tradition grammaticale prescriptive française persiste à considérer le système de genre grammatical comme un système arbitraire, fonctionnant de manière identique pour les noms inanimés et pour les humains. Selon cette idée le masculin peut endosser une fonction de référence «neutre » ou " générique ». En s'appuyant sur l'analyse du linguiste structuraliste Jakobson (1939) pour le russe, l'Académie Française $(1984,2014)$ expliquait ${ }^{1}$ :

$\mathrm{Si}$, en effet, le français connaît deux genres, appelés masculin et féminin, il serait plus juste de les nommer genre marqué et genre non marqué. Seul le genre masculin, non marqué, peut représenter aussi bien les éléments masculins que féminins. En effet, le genre féminin ou marqué est privatif: un "groupe d'étudiantes » ne pourra contenir d'élèves de sexe masculin, tandis qu'un « groupe d'étudiants " pourra contenir des élèves des deux sexes, indifféremment. On se gardera également de dire les électeurs et les électrices, les informaticiennes et les informaticiens, expressions qui sont non seulement lourdes mais aussi redondantes, les informaticiennes étant comprises dans les informaticiens.

Pareillement, les grammairiens Grévisse \& Goose (2008:619) proposent que :

Le masculin, étant le genre indifférencié, s'emploie aussi sans s'opposer au féminin pour désigner des personnes, ou une personne, en faisant abstraction de leur sexe ou de son sexe ; cela arrive aussi, mais plus rarement pour le féminin.

Pour celles et ceux qui adoptent cette perspective, le biais favorisant les hommes serait simplement extra-linguistique car lié aux stéréotypes de genre. Par exemple, Claude Hagège explique que «la confusion du genre grammatical avec le sexe a de vieilles et solides assises » et que :

Certes, la langue porte des marques de la domination masculine. Mais ce n'est pas langue elle-même qui est sexiste. Ceux qui le sont, ce sont les hommes. En outre, on peut considérer le masculin, quand il commande l'accord, non comme un masculin réel, mais comme une forme neutre (les linguistes disent " non marquée »), c'est-àdire associant les deux genres. [...] Ce n'est pas l'intervention sur la langue qui transformera les comportements sociaux. C'est l'évolution des comportements sociaux qui s'inscrira dans la langue (Hagège 2017:1-2²).

Cette position est contestée cependant tant par la littérature féministe (e.g. HoudebineGravaud, 1995 ; Khaznadar, 2007 ; Baider, Khaznadar \& Moreau, 2007 ; Michard, 1996, 2001; Michel, 2016) que par de récents travaux en psycholinguistique (e.g. Chatard et al., 2005 ; Brauer \& Landry, 2008 ; Gabriel, et al 2008 ; Garnham, et al., 2012 ; Gygax et al., 2019). Dans cet article, nous présentons un nouvel élément renforçant l'argument de ces deux courants de recherche : le genre grammatical masculin en français crée un biais masculin dans l'interprétation de syntagmes nominaux référant aux humains allant au-delà des stéréotypes, qui pourtant jouent aussi un rôle. Notre argumentation se base sur un nouveau paradigme d'expériences psycholinguistiques qui oppose l'interprétation des phrases françaises où le marquage de genre est visible (comme (3a) et (3b)) avec des phrases où le marquage est neutralisé grâce à son contexte morphophonologique (3c). 
(3) a. Le journaliste a appelé.

b. La journaliste a appelé.

c. L'incroyable journaliste a appelé.

7 De plus, nos expériences fournissent une nouvelle façon de quantifier la contribution $\mathrm{du}$ marquage de genre par rapport aux croyances des locutrices et locuteurs francophones, nous permettant ainsi d'observer plus finement les rapports entre langage, idéologies et discours genrés en français.

8 L'article s'articule comme suit: en section 2, nous faisons un bref état de l'art impliquant les expériences psycholinguistiques investiguant le genre grammatical en français et les stéréotypes de genre. En section 3, nous présentons notre première expérience : une expérience d'estimation de croyances (belief estimation) qui porte sur des phrases où le genre grammatical est neutralisé (eg. (3c)). Ensuite, dans la section 4, nous présentons les résultats d'une expérience d'estimation de croyances parallèle qui implique des phrases comme $(3 a, b)$ où le genre grammatical est visiblement marqué. Enfin, nous discutons des implications de nos résultats, notamment sous le prisme de l'androcentrisme (Bodine, 1975 ; Spender, 1980 ; Bem, 1993) et en termes de politique de langage non discriminant.

\section{Travaux sur le genre grammatical et les représentations mentales}

9 Nos recherches se situent dans le domaine de la psycholinguistique. Il est d'abord important de noter qu'il existe une tradition de recherche riche (et croissante) sur le genre grammatical et les croyances des locutrices et locuteurs francophones dans ce domaine. Cette tradition repose en partie sur des travaux sur les stéréotypes de genre et le langage en anglais et enrichit nos connaissances en incorporant le genre grammatical aux analyses. Une des premières études psycholinguistiques à s'être penchée sur la question de la non-neutralité interprétative du genre grammatical français est celle de Brauer et Landry (2008). Ils ont testé l'influence du genre grammatical sur les représentations mentales des locutrices et locuteurs du français de plusieurs classes d'âges (enfants, étudiants, adultes) à travers cinq études. Dans les cinq expériences, les participantes et participants étaient exposés soit à une forme masculine comme en (4a) soit à une forme inclusive comme en (4b).

(4) a. Sans tenir compte de vos opinions politiques, citez tous les candidats de droite que vous verriez au poste de Premier ministre.

b. Sans tenir compte de vos opinions politiques, citez tous les candidats/ candidates de droite que vous verriez au poste de Premier ministre

10 Dans la première étude, les participantes et participants devaient citer des responsables politiques (comme en (4)). La deuxième étude demandait d'identifier les héros historiques et autres figures publiques (e.g. « vos héros dans la réalité » vs « vos héros/hérö̈nes dans la réalité »), quand la troisième demandait d'imaginer et décrire la personne représentant typiquement une profession (e.g. apparence physique, trait de caractère et passe-temps, etc, de " avocats » vs apparence physique, trait de caractère et passe-temps de "avocats/avocates». Dans la quatrième étude, des enfants en école primaire devaient dessiner une personne exerçant une profession donnée (e.g. « sais-tu 
ce que font les avocats? Dessine-nous, sur cette page, une personne en train de faire ce métier » vs " "sais-tu ce que font les avocats/avocates ?"). Enfin, la cinquième étude consistait en une tâche de lecture à propos d'un congrès professionnel. Les participantes et participants devaient, deux heures après avoir lu le texte, donner le pourcentage d'hommes et de femmes participant à ce congrès, bien qu'un tel chiffre ne soit en réalité pas précisé dans le texte. Ils ont ainsi montré que le genre masculin restreignait l'interprétation à sa fonction de genre spécifique : les groupes exposés aux formes inclusives (ex: les candidats/candidates) référaient plus souvent à des femmes que les groupes exposés seulement aux formes masculines (e.g. candidats). Cette étude montre par ailleurs que le biais créé par le genre masculin s'étend également à l'épicène ${ }^{3}$ individu. Bien que, par son caractère épicène, ce nom puisse référer à tout type d'humains, de genre grammatical masculin, il favorise aussi une représentation mentale masculine. À l'inverse, l'épicène féminin personne ne déclenche aucun biais : ni en faveur des hommes ni en faveur des femmes.

11 Les études sur l'anglais, une langue où le genre grammatical existe uniquement sur les pronoms, ont démontré que des expressions qui auraient une dénotation sémantique non genrée peuvent quand même acquérir une interprétation biaisée en faveur du masculin, selon le contexte, les croyances et les idéologies des locuteurs et locutrices. Suivant une approche empirique, Cameron $(1985,2018)$ défend l'idée selon laquelle, en anglais, l'utilisation de syntagmes nominaux formellement neutres n'implique pas nécessairement que la référence soit inclusive. Elle souligne que ces substantifs, non marqués en genre, font en fait souvent référence spécifiquement aux hommes, excluant par là même les femmes (et toutes les autres personnes non binaires). Elle dit (Cameron 1985 : 84-85) :

Because spaceman incorporates the word man, whose meaning has narrowed (become more specialised) from meaning 'person' to meaning 'male person', it strongly suggests a male referent. The implication of spaceman is that women cannot fly rockets, walk on the moon, etc. But once the linguistically marked male element man is removed, the argument runs, people will not think male any more. The possibility will exist that women can fulfil the new role, non-sexistly designated astronaut.

But what if the word astronaut, despite having no overt markers of maleness, is used by most people as if it too were male only? There is a large amount of evidence that this is in fact what happens with words that are not linguistically gendermarked. Consider, for example, the following extracts from newspapers:

The lack of vitality is aggravated by the fact that there are so few able-bodied young adults around. They have all gone off to work or look for work, leaving behind the old, the disabled, the women and the children. The Sunday Times

A coloured South African who was subjected to racial abuse by his neighbours went berserk with a machete and killed his nextdoor neighbour's wife, Birmingham Crown Court heard yesterday. The Guardian

In these examples, two phrases without overt linguistic marking of gender are used as if they could only be applied to men: able-bodied young adult and next-door neighbour. In the first case, the disabled, the old and the non-adult are clearly excluded by the meaning of the words which make up the phrase. But why are women excluded? In the second case, since apparently the murdered woman lived 
next door to her attacker, why was she not 'the next door neighbour' but just 'the next door neighbour's wife?

12 Selon Cameron, ce qui se passe avec astronaut, able-bodied young adults et neighbour est que les locutrices et locuteurs anglophones - au moins certain·e's - associent une représentation fortement masculine à ces expressions. Autrement dit, grâce à notre expérience et notre culture androcentrique, des noms comme astronaut et même des noms comme adults ou neighbour ont des stéréotypes masculins.

13 La question des stéréotypes de genre dans les syntagmes nominaux en anglais a été investiguée avec des méthodes psycholinguistiques par Carreiras et al. (1996), Garnham et al. (2002), Misersky et al. (2014) et Garnham et al. (2015), entre autres. Misersky et al. ont développé un score de stéréotypicalité pour 422 noms de métiers ou d'occupations dans plusieurs langues dont l'anglais et le français. Les scores de cette étude représentent la croyance des participantes et participants quant à la proportion d'hommes et de femmes pour chaque corps de métier/occupation. Les noms étaient présentés sous les deux formes pour les langues à genre grammatical (masculine et féminine) et les mentions « homme » et «femme » étaient spécifiées pour les langues sans genre. Les participantes et participants devaient ensuite donner sur une échelle à onze niveaux ce qui leur semblait être la réelle proportion de femmes et d'hommes pour chaque occupation/rôle. Les scores reportant la stéréotypicalité des noms vont de 0 à 1,0 représentant une occupation $100 \%$ masculine et $1,100 \%$ féminine.

Ensuite, Garnham et al. (2015) ont comparé les résultats de Miserksy et al., qui portent sur les croyances des locutrices et locuteurs anglophones sur la proportion d'hommes/ femmes exerçant un métier, avec les proportions statistiques fournies par le UK Office for National Statistics et d'autres sources. Le mapping entre les 422 noms testés par Miserksy et al. et les noms de métiers recensés par le UK Office for National Statistics n'était pas toujours parfait. Cependant, Garnham et al. ont pu mettre en relation le score Misersky et les proportions observées de 290 noms de métiers. Ils ont trouvé qu'il existe une corrélation statistiquement significative forte entre la proportion hommes/ femmes exerçant un métier et les croyances des locutrices et locuteurs à propos de cette proportion (i.e. le score Misersky). Cette corrélation est représentée en figure 1. 
Figure 1 : Corrélation entre le ratio femmes/hommes publié par le UK Office for National Statistics et le score Misersky selon l'étude de Garnham et al. 2015.

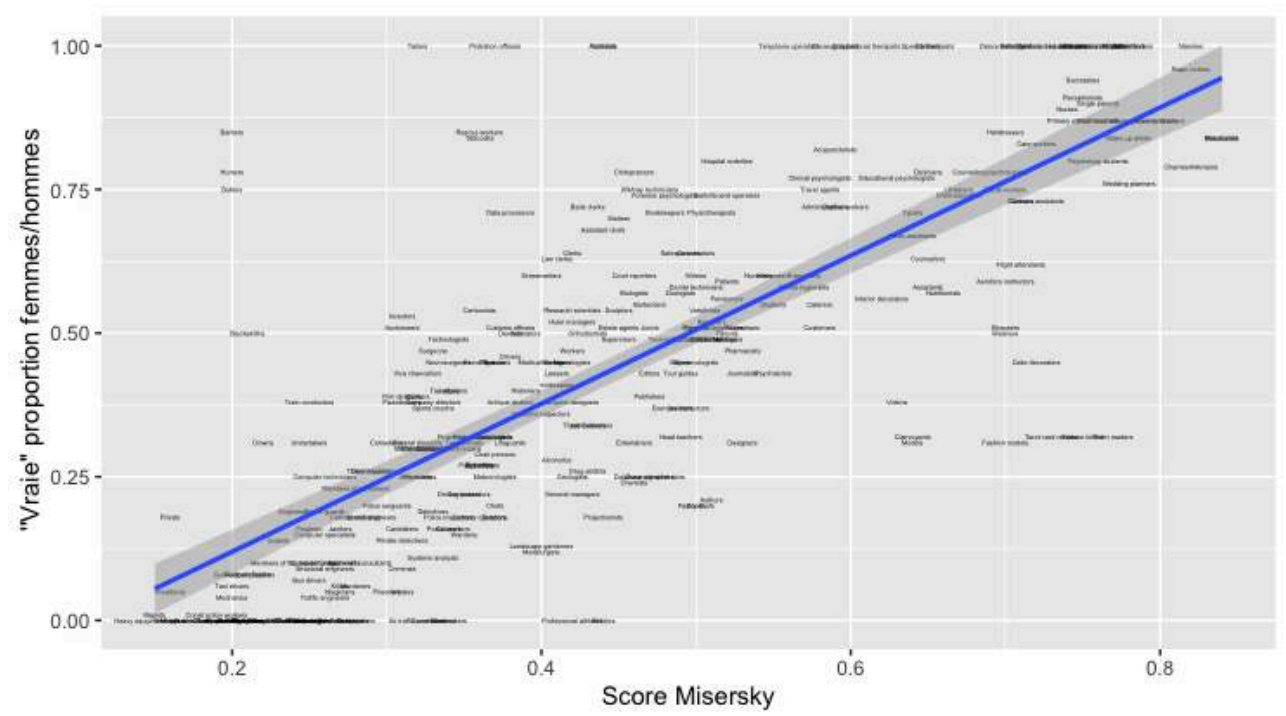

Comme décrivent Garnham et al., les scores Misersky et les proportions statistiques sont très proches, et, en fait, les scores Misersky sont mêmes moins extrêmes/ stéréotypés que la réalité. Ces chercheurees concluent alors que, à quelques exceptions près (voir Garnham et al. pour plus de précisions), de manière générale les stéréotypes de genre associés aux noms de métiers en anglais (une langue sans genre grammatical, excepté pour les pronoms) ont une influence sur l'interprétation des noms et, de plus, il existe un lien entre ces stéréotypes et les inégalités de genre au travail (voir aussi Gygax et al. 2016).

L'idée que les stéréotypes peuvent contribuer à une interprétation biaisée d'expressions linguistiques a aussi été proposée pour le français. Dans une étude du discours genré dans les travaux d'anthropologues, Michard $(1999,2019)$ montre entre autres que les noms collectifs utilisés par les anthropologues (e.g. société, tribu, village (5)), cités de Michard (2019:182)) peuvent avoir un biais androcentrique et référer en réalité aux hommes qui composent ces groupes. Les femmes sont quant à elles l'objet d'une description spécifique, et ne sont alors pas incluses dans ces noms collectifs qui ont pourtant comme caractéristique première de référer à un ensemble d'êtres humains.

(5) a. Il faut en effet souligner que, pour les Indiens, l'obligation de donner le gibier n'est nullement vécue comme telle, tandis que celle de partager l'épouse est éprouvée comme aliénation [XIV] (Clastres $1966: 26)$.

b. Même si l'enfant est mort-né, les Baruya soupçonnent toujours leur femme d'avoir tué leur enfant [XV] (Godelier 1976 : 285).

c. Le village entier partit le lendemain dans une trentaine de pirogues, nous laissant seuls avec les femmes et les enfants dans les maisons abandonnées [XVI] (Lévi-Strauss 1936 : 283).

Le biais masculin des noms collectifs rapproche dès lors le français de l'anglais, langue presque dépourvue de genre grammatical.

Ceci dit, la littérature psycholinguistique sur le français présente des résultats variés quant à l'importance relative des stéréotypes et le genre grammatical dans l'interprétation genrée. Par exemple, Gygax et al. (2008) ont étudié les effets combinés 
des stéréotypes sociaux et du genre grammatical masculin sur les représentations mentales des locutrices et locuteurs de l'anglais, de l'allemand, et du français. Pour chacune de ces trois langues, des paires de phrases étaient présentées aux participantes et participants qui devaient ensuite décider si la seconde phrase (6b) était une continuation possible de la première (6a). Ces continuations précisaient que le groupe était composé au moins de quelques femmes (ex: plusieurs femmes) ou de quelques hommes (ex : plusieurs hommes). Les noms (6a) étaient systématiquement marqués du genre masculin pluriel pour le français et l'allemand, et seulement du pluriel pour l'anglais, langue sans genre grammatical à l'exception des pronoms. Les noms de rôles ou de métiers choisis étaient soit stéréotypiquement masculins, stéréotypiquement neutres ou stéréotypiquement féminins.

(6) a. Les assistants sociaux marchaient dans la gare.

b. Du beau temps étant prévu, plusieurs femmes n'avaient pas de veste.

19 En anglais, en lien avec les travaux cités ci-dessus, seuls les stéréotypes avaient un effet sur le type de réponse donné. A l'inverse, en français et en allemand, seul l'effet du genre grammatical a permis de prédire le type de réponse des participantes et participants : plus de réponses positives étaient données lorsque la seconde phrase précisait que le groupe était au moins composé d'hommes, mais il n'y avait ni un effet significatif du stéréotype (féminin, neutre ou masculin) ni de l'interaction entre le genre grammatical et le stéréotype. Par ailleurs, lorsque les participants et participantes répondaient positivement à une continuation féminine, les temps de réaction étaient significativement plus longs que pour les continuations masculines, soulignant dès lors la difficulté d'accéder à l'interprétation générique du masculin. Cette étude semblerait démontrer que les effets du genre grammatical surpassent largement ceux de l'activation potentielle des stéréotypes de genre.

Ceci dit, Garnham et al. (2012) ont fait une expérience suivante où ils ont pris les stimuli de Gygax et al. (2008) et ont ajouté une deuxième phrase avec un pronom qui référait au sujet de la première phrase (7a). Comme Gygax et al. (2008), Garnham et al. ont ensuite demandé aux participantes et participants si la phase (7b) était une bonne continuation de la première (7a).

(7) a. Les voisins sortirent de la cafétéria. Ils partirent.

b. À cause du temps nuageux, un[e] des femmes [hommes] avait un parapluie.

21 Cette fois ci, Garnham et al. ont trouvé, en plus d'un effet du genre grammatical, un effet de stéréotype : la différence dans l'acceptabilité des continuations référant à des hommes avec le pronom ils était significativement plus grande quand le sujet comportait un nom avec un stéréotype masculin (27\%) que quand le sujet avait un nom avec un stéréotype féminin (16\%) ou neutre (14\%). D'autres études sur le français et l'allemand (Irmen, \& Kurovskaja, 2010 ; Gygax et al. 2019, Vervecken et al. 2015) vont dans ce sens : les stéréotypes et le genre grammatical semblent interagir.

$\mathrm{Vu}$ ces résultats divers, plus d'études psycholinguistiques sur la (non)interaction entre le genre grammatical et les stéréotypes seraient souhaitables. De plus, les études de Gygax et al. (2008) et Garnham et al. (2012) ont porté sur l'interprétation genrée des pluriels (les assistants sociaux, les voisins etc.). À notre connaissance, à l'exception de Rothermun (1998) sur l'allemand, la question de si/comment les stéréotypes et le 
marquage de genre interagissent chez les singuliers, n'a pas jusqu'à présent été étudiée pour le français. Finalement, afin de pouvoir démêler les effets de stéréotypes et de genre grammatical, Gygax et al. (2008) avaient comparé le français (et l'allemand) directement avec anglais. Ils disent $(2008: 470)$ :

In this paper, we investigated these issues by directly assessing the interaction between stereotype and masculine biases in German and French, which we compared to English that should establish a baseline for a stereotype bias only, as it is difficult or impossible to construct a relevant control condition in German and French in which stereotype biases act alone, without the effects of morphological gender marking.

Comme nous avons expliqué ci-dessus, la comparaison avec l'anglais est éclairante, mais il n'est pas tout à fait vrai qu'il est impossible de construire des conditions de " contrôle» sans marquage de genre grammatical en français. Comme nous avons montré en (3), il existe certains contextes, comme des syntagmes nominaux avec des adjectifs préverbaux à voyelle initiale, où le marquage de genre n'est pas visible. Par conséquent, nous avons testé l'interprétation d'un ensemble identique de trente-six noms selon le marquage ou l'absence de genre grammatical, que nous décrivons maintenant. Compte tenu de ce qui est observé en anglais, nous prédisons que l'interprétation genrée des syntagmes neutralisés sera influencée par les stéréotypes. Pour les syntagmes avec marquage de genre, soit l'interprétation du genre masculin va «noyer » l'effet de stéréotype, comme dans Gygax (2008), soit l'effet du stéréotype survivra, comme dans Garnham (2012).

\section{Expérience 1 : Neutralisation du genre}

\section{Stimuli et procédure}

Trente-six noms de métiers de type common gender (Corbett 1991) issus de l'étude de Misersky et al. (2014), soit douze stéréotypiquement masculins, douze stéréotypiquement féminins et douze stéréotypiquement neutres, ont été testés ${ }^{4}$.

Lorsque plusieurs genres grammaticaux peuvent être assignés à un même nom comme en (8), résultant dès lors en un changement sémantique, Corbett $(1991: 67,181)$ désigne ce phénomène de common gender. Il ne s'agit pas d'un nouveau type de genre grammatical mais plutôt d'une autre manière pour le nom de déclencher un accord syntaxique cohérent en genre sur tous ses dépendants.

(8). a. La veille d'un spectacle, le violoniste talentueux a toujours le trac.

b. La veille d'un spectacle, la violoniste talentueuse a toujours le trac.

Ici, violoniste peut déclencher un accord masculin ou féminin sur ses dépendants (le déterminant et l'adjectif) sans rendre l'énoncé agrammatical ou inacceptable. Nous pouvons dès lors opposer le comportement d'un nom comme violoniste à celui d'un nom tel que boulanger. Dans le second cas, boulanger n'est compatible qu'avec le genre grammatical masculin, et n'est donc pas un nom de type common gender (i.e. *la boulanger talentueuse).

Pour neutraliser la marque du genre grammatical, nous avons utilisé trente-six adjectifs, également de type common gender, commençant tous par une voyelle. Les adjectifs devaient systématiquement être antéposés au nom qu'ils qualifiaient pour 
faire tomber le genre grammatical (9) - qui, dans notre seconde expérience, n'est marqué que par un déterminant.

(9) La veille d'un spectacle, l'unique violoniste a toujours le trac. n'ajoutait pas de biais sur leur interprétation, nous avons constitué douze groupes composés chacun des trois types de stéréotypes - nom « masculin ", nom "féminin ", nom « neutre » - pour lesquels trois contextes leur étaient associés. La classification de noms par stéréotypicalité suit le score de Misersky et al. (2014) pour le français. Par ailleurs, pour contrebalancer les effets potentiels des adjectifs sur l'interprétation des noms, nous avons utilisé trois adjectifs pour chaque groupe de trois noms (3 adjectifs $\mathrm{x}$ 3 noms $x 3$ contextes).

Nous avons procédé de la même manière pour les deux expériences. L'attribution des items a été faite de manière aléatoire sur Ibex. Chaque participantee a été confrontée aux trente-six noms mais n'a jamais vu deux fois la même phrase de contexte ou le même adjectif. Quarante fillers ${ }^{5}$ ont été ajoutés à l'expérience. Il s'agissait de prénoms épicènes insérés dans diverses phrases descriptives du même ordre que les phrases items (10).

(10) Le dimanche matin, Camille aime aller courir au parc.

Nous avons diffusé cette expérience sur les réseaux sociaux, par conséquent nous n'avons opéré aucun contrôle sur les facteurs sociaux liés aux sujets. Les trente-six items ainsi que les quarante phrases fillers ont été présentées aux participantes et participants de la façon suivante :

Figure 2 : Procédure

Quand il pleut, I'aimable graphiste sort avec son parapluie

A votre avis, cette personne est plutôt:

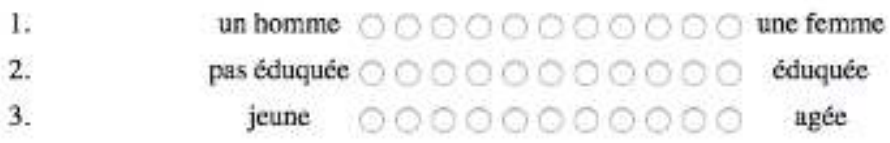

31 Afin de limiter notre influence sur les réponses des participantes et participants, nous avons utilisé une échelle à 11 niveaux $^{6}$ (allant de 0 à 1 ), représentant au mieux un continuum pour les trois catégories choisies (genre, niveau d'éducation, âge). Une échelle à onze niveaux permet un meilleur parallèle avec les scores de stéréotypicalité de Misersky et al. (2014). Nous avions précisé que chaque cercle le plus proche de chaque catégorie représentait le plus cette catégorie, et que le cercle du centre correspondait à "neutre ». Le niveau d'éducation et l'âge constituaient aussi des distracteurs, pour que le thème précis de notre recherche soit le moins évident possible à déterminer. Dans la mesure où Brauer et Landry (2008) ont démontré la neutralité du terme personne (à opposer à individu), nous avons exclusivement utilisé cet épicène. 


\section{Analyses statistiques} influencer les locuteurs et locutrices dans leur choix pour déterminer le genre social d'un référent. Il est donc possible que certains facteurs sociaux comme l'âge des participantes et participants, leur genre et leur type d'études influencent leur perception du genre grammatical, ou du moins leur perception du genre grammatical en combinaison avec les stéréotypes sociaux. Concernant l'âge, les personnes n'évoluant pas selon les mêmes dynamiques et problématiques sociales ont un rapport différent aux formes standards de langue (Eckert, 1998; Labov, 2001). Selon Labov (2001 : 101) les plus jeunes ne sont pas encore insérées complètement dans la société, à opposer par exemple aux 30-59 ans qui sont généralement engagées dans leur carrière professionnelle et/ou une vie familiale, entretenant par conséquent un rapport aux normes langagières différent des plus jeunes. De la même façon, les retraités peuvent avoir un registre de langue plus relâché car leurs rapports aux normes sociales et langagières ne sont plus en lien direct avec leur socialisation (éducation des enfants et carrière professionnelle). Nous avons alors constitué trois macro-catégories pour l'âge : les participantes et participants les plus jeunes (18-25), les âges médians (26-50) et les plus âgées (51 et plus). Ainsi, nous avons voulu prédire les scores d'interprétation à l'aide d'un modèle linéaire mixte sur R (R Core Team, 2020) en utilisant le package lme4 (Bates et al., 2015). Nous avons utilisé les participantes, participants et les items comme facteurs aléatoires, et les facteurs sociaux (âge, genre et type d'études), ainsi que les stéréotypes associés aux métiers comme prédicteurs.

\section{Résultats et discussion}

Comme le montre la figure 4, en l'absence de marquage du genre grammatical, les participantes et participants semblent effectivement se fier aux stéréotypes de genre pour évaluer le genre du référent. Les noms stéréotypiquement masculins ont obtenu en moyenne un score de .30 (écart type $=.24$ ), les noms neutres ont obtenu un score moyen de .46 (écart type $=.26$ ) et les noms féminins un score moyen de .61 (écart type $=$ .27). De plus, la relation étroite entre le score Misersky et le score d'interprétation obtenu ici apporte un argument en faveur de l'utilisation de cette mesure de stéréotypicalité au français hexagonal. En effet, cet outil a d'abord été développé d'abord pour les locutrices et locuteurs francophones suisses. C'est d'ailleurs certainement pour cette raison que logopédiste (orthophoniste en France) est un « outlier» dans nos données (voir la figure 3). 
Figure 3 : scores moyens d'interprétation en fonction des scores Misersky

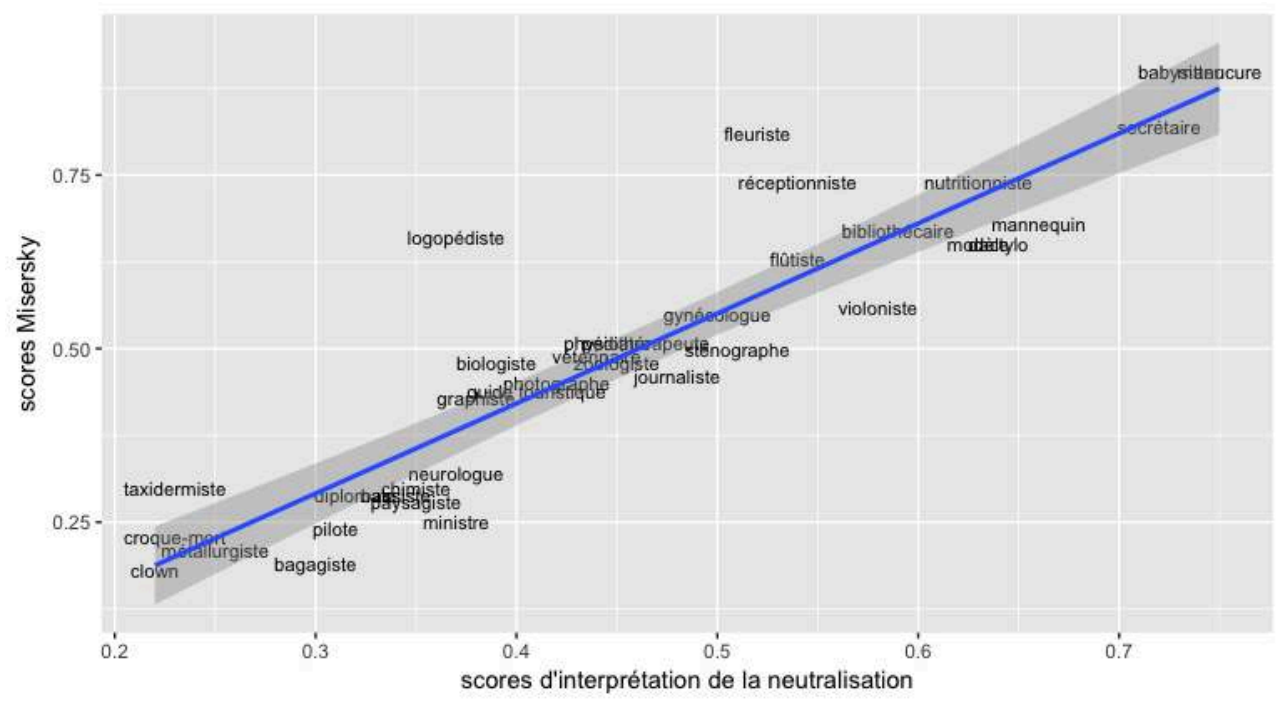

Pour les analyses statistiques : en utilisant un modèle linéaire où les participantes et participants et les items sont inclus comme facteurs aléatoires, les scores Misersky prédisent bel et bien les scores obtenus lors de cette expérience: plus les scores Misersky sont élevés, plus il y a de chance que le nom soit perçu comme référant à une femme (tableau 1). Il faut aussi souligner qu'aucun facteur social n'a eu d'effet sur l'interprétation du genre grammatical: ni l'âge, ni le genre des participantes et participants.

Tableau 1 : effet fixe du modèle linéaire (l'effet des variables indépendantes sur notre variable dépendante).

\begin{tabular}{|l|l|l|l|l|l|l|}
\hline & Estimate & Std. Error & $\mathrm{df}$ & $\mathrm{t}$ value & $\operatorname{Pr}(>|\mathrm{t}|)$ & \\
\hline (Intercept) & 0.146877 & 0.029137 & 47.913310 & 5.041 & $7.04 \mathrm{e}-06$ & $* * *$ \\
\hline Scores Misersky. & 0.639812 & 0.049230 & 34.001696 & 612.997 & $9.57 \mathrm{e}-15$ & $* * *$ \\
\hline+50 ans & 0.011119 & 0.023984 & 42.995714 & 0.464 & 0.645 & \\
\hline $18-25$ ans & -0.016562 & 0.017816 & 42.995714 & -0.930 & 0.358 & \\
\hline Hommes & 0.007073 & 0.022022 & 42.995714 & 0.321 & 0.750 & \\
\hline Genre autre & -0.090933 & 0.055520 & 42.995714 & -1.638 & 0.109 & \\
\hline
\end{tabular}


Figure 4 : régression linéaire de l'interprétation des noms lorsque le genre grammatical est neutralisé.

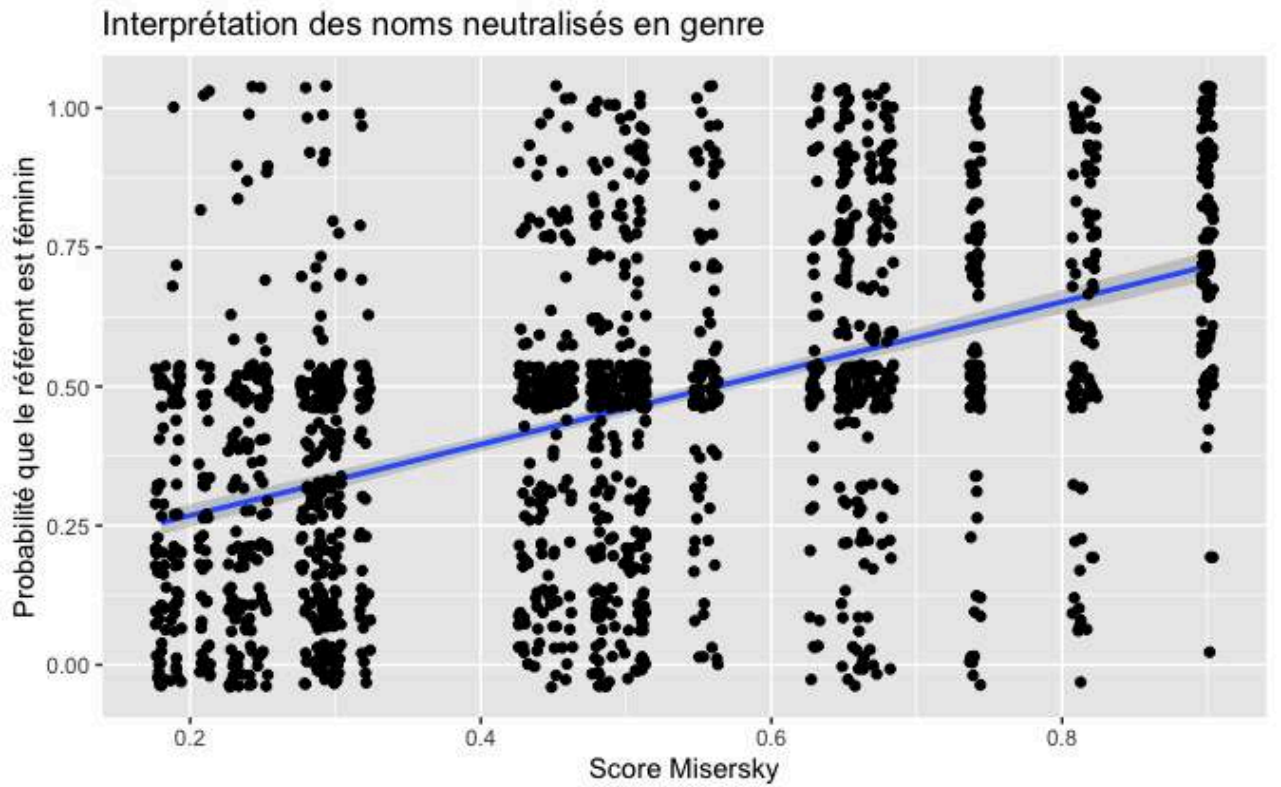

La régression linéaire permet de créer un modèle du type de réponse obtenue (ici la probabilité que le référent soit féminin) en fonction de la variable explicative/ indépendante (ici le score Misersky). Ainsi, en résumé, lorsque le genre grammatical est complètement neutralisé, les locuteurs et locutrices du français (de France) interprètent les syntagmes comme les locuteurs et locutrices anglophones (Boyce et al., 2019) : les construits sociaux sont dans ce cas une ressource disponible pour déterminer le genre du référent. Notre modèle ne montre aucun effet de l'âge sur le type d'interprétation des noms. En d'autres termes, l'effet des stéréotypes est constant à travers les différentes classes d'âges observées.

De plus, comme Garnham et al. (2015), nous observons que les scores d'interprétation de syntagmes neutralisés sont fortement corrélés avec les proportions d'hommes et de femmes dans les professions différentes recensées par l'INSEE, comme le montre la figure 5. Il faut bien noter que les catégories d'INSEE qui sont publiquement disponibles sont beaucoup moins raffinées que nos catégories, qui viennent de Misersky et al. 2014. Par exemple, fleuriste, qui a un score Misersky de 0.81 (donc stéréotype fortement féminin), est inclus dans la catégorie d'INSEE Commerçants et assimilés qui, en tout, a une proportion de femmes de 0.32. Donc il est raisonnable de supposer que la corrélation entre les jugements des participantes et participants dans un contexte neutralisé et les ratios hommes/femmes observés est encore plus étroite que la suggère la figure 5. 
Figure 5 : Corrélation entre le ratio hommes/femmes observé et le score d'interprétation, genre grammatical neutralisé.

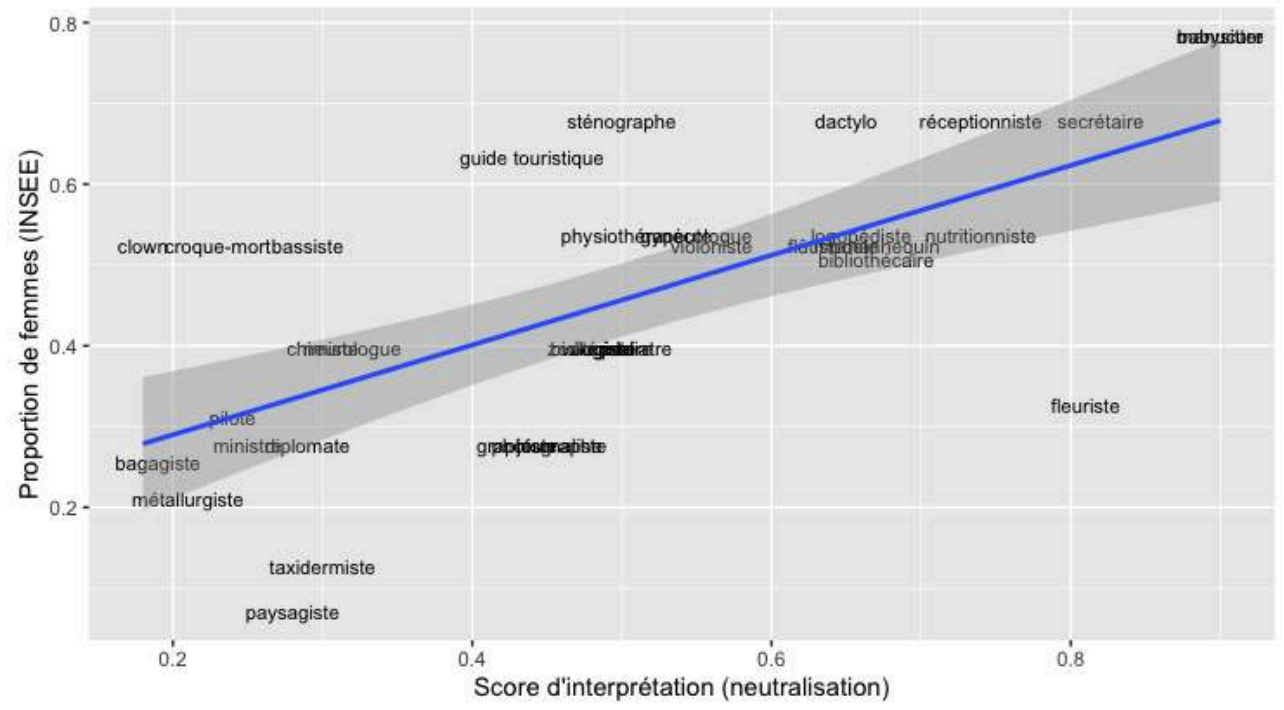

Ces résultats sur l'interprétation des syntagmes nominaux dont le marquage de genre est neutralisé nous permettent, dans un premier temps, d'observer de parallèles proches entre l'anglais et le français : l'interprétation des syntagmes nominaux où le genre grammatical est neutralisé est influencée par les stéréotypes. Dans un deuxième temps, ces résultats nous permettent aussi d'observer que les croyances des locuteurs et locutrices dans des contextes de neutralisation du genre grammatical sont assez bien calibrées avec les proportions de genre social données par l'INSEE.

Comme nous allons voir dans la prochaine section, ceci ne sera plus le cas, une fois qu'on ajoute le genre grammatical.

\section{Expérience 2 : Marquage du genre}

\section{Stimuli et procédure}

41 Nous avons testé les trente-six noms common gender dans des phrases où le genre grammatical était marqué sur le déterminant (le ou la). Comme dans l'expérience de neutralisation, afin de nous assurer que chaque énoncé dans lequel se trouvaient les items testés n'ajoutait pas de biais sur leur interprétation, nous avons repris les douze groupes composés chacun des trois types de stéréotypes - nom "masculin », nom " féminin », nom «neutre " - pour lesquels trois contextes leur étaient associés. De la même manière, les deux genres grammaticaux apparaissaient avec tous les noms dans tous les contextes, comme en (11) et (12). Au total, il y a donc autant de contextes que de noms, soit trente-six, et chaque participante ne voyait qu'un seul contexte associé à un nom et un genre grammatical ( 2 genres gram $x 3$ noms $x 3$ contextes).

(11) a. Pour téléphoner, le/la diplomate/secrétaire/journaliste appelle toujours en masqué.

b. Quand son travail est fini, le/la diplomate/secrétaire/journaliste fait toujours une sauvegarde.

c. Si l'ordinateur est cassé, le/la diplomate/secrétaire/journaliste note tout dans son agenda. 
(12) a. Pendant les vacances d'hiver, la/le ministre/réceptionniste/graphiste part au ski.

b. Pour l'heure du déjeuner, la/le ministre/réceptionniste/graphiste mange un sandwich.

c. Quand il pleut, la/le ministre/réceptionniste/graphiste sort avec son parapluie. L'attribution des items a été faite de manière aléatoire sur Ibex, tout en conservant un ordre précis (participante 1 voyait tous les premiers types items, participante 2 les seconds, et ainsi de suite, puis participante 7 voyait à nouveau les premiers items, etc.). Par ailleurs, pour éviter que la variation en genre n'influence trop les participantes et participants et qu'ils et elles ne répondent de manière mécanique et binaire, dix-huit noms leur étaient d'abord présentés accompagnés du déterminant masculin, puis dixhuit autres accompagnés du déterminant féminin. L'idée derrière cette stratégie était la suivante: s'il est possible que le masculin déclenche une interprétation neutre en genre, cette dernière sera favorisée dans ce contexte plutôt qu'en alternant aléatoirement le et $l a^{8}$. Gygax et Gabriel (2008) avaient également montré que l'alternance de référents masculins et féminins renforçait l'interprétation spécifique du masculin. Enfin, les quarante fillers (phrases avec prénoms épicènes) de l'expérience précédente ont été ajoutés à l'expérience.

Nous avons diffusé cette expérience sur les réseaux sociaux. Les trente-six items ainsi que les quarante phrases fillers ont été présentées aux participantes et participants de la façon suivante :

Figure 6 : Procédure

Quand il y a les jeux olympiques, le vétérinaire regarde la télévision.

A votre avis, cette personne est plutôt:

1.

un homme

2.

3. une femme

éduquée

agée

Soixante-deux personnes ont participé à l'expérience, mais une participante a été écartée de l'analyse parce qu'elle n'était pas locutrice native du français.

\section{Résultats}

\section{Description des données}

Les scores ci-dessous (Figure 7 et 8), représentent les scores moyens pour chaque nom selon le genre grammatical affilié (en abscisse). Ils ont été arrondis au centième près de manière à faciliter la comparaison avec les scores de stéréotypicalité (Misersky et al., 2014) représentés en ordonnée. 
Figure 7 : Scores d'interprétation pour le genre masculin en fonction des scores Misersky et al. (x va de 0.1 à 0.6 )

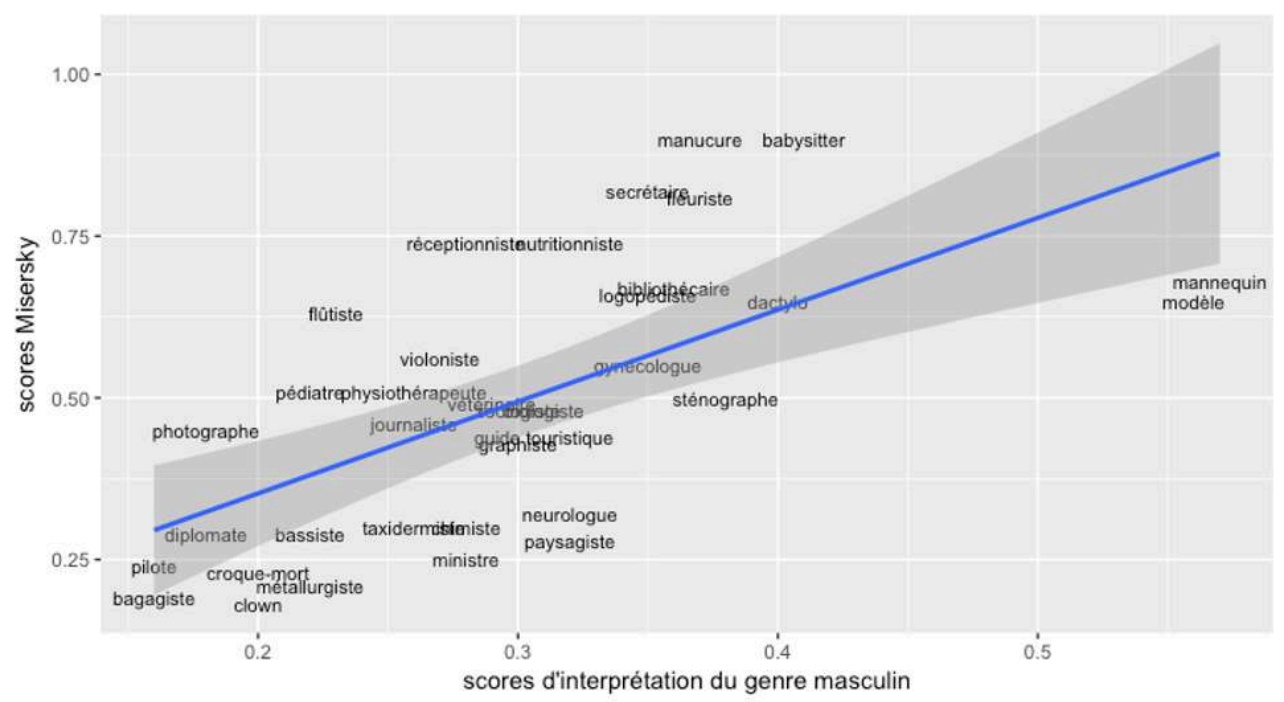

Figure 8 : Scores (moyennes) d'interprétation pour le genre féminin en fonction des scores Misersky et al. ( $x$ va de 0.65 à 1$)$

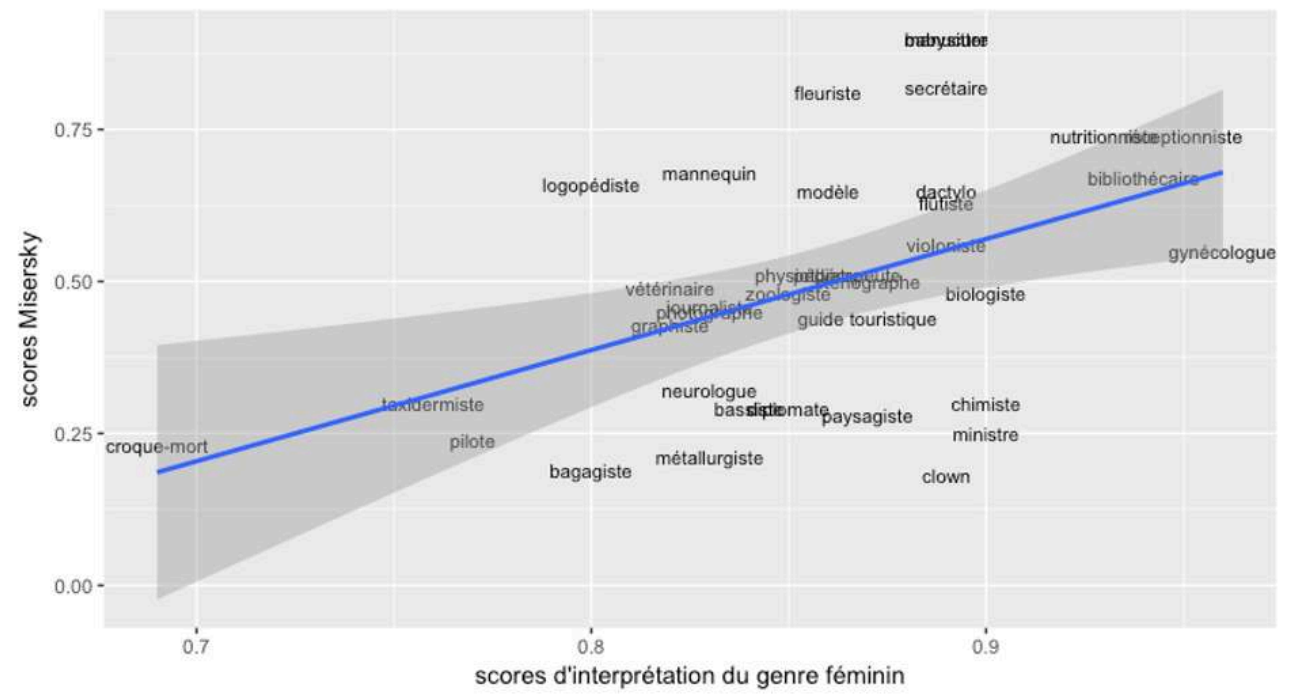

En premier lieu, nous pouvons constater qu'effectivement, les scores diffèrent entre le masculin et le féminin. Accompagnés du déterminant la, les noms s'étalent sans grande surprise de 0.69 (la croque-mort) à 0.96 (la gynécologue). Avec le déterminant le, les noms vont de 0.16 (le pilote, le bagagiste) à 0.57 (le mannequin). De ce fait, comme l'ont montré Gygax et al. (2008), le genre grammatical semble bien être le premier facteur permettant aux locuteurs et locutrices de déterminer le genre des individus référés. Ceci est particulièrement remarquable pour les noms «neutres » qui permettent moins aux participantes et participants de s'appuyer sur un facteur externe saillant pour déterminer le genre de la personne, et donc seraient plus à même de favoriser une interprétation "générique/neutre» du genre grammatical masculin. Cela n'est pourtant pas le cas ici puisque ces noms ne sont pas traités différemment des autres $(\mathrm{MIN}=0.18$ le photographe $; \mathrm{MAX}=0.38$ le sténographe $)$. 
Cependant, comme l'ont montré Garnham et al. (2012), nous avons trouvé des différences de moyennes générales par stéréotype. En moyenne, les noms stéréotypiquement masculins ont des scores plus bas que les noms dits neutres, euxmêmes plus bas que les noms stéréotypiquement féminins. Ainsi, les stéréotypes paraissent moduler les effets majeurs du genre grammatical. Nous discutons en détail de ce point lors de nos analyses statistiques ci-dessous.

Enfin, seuls trois noms stéréotypiquement féminins - modèle, mannequin et babysitter ont obtenu des scores pouvant montrer une lecture non biaisée du genre masculin. Mais il faut souligner la différence de scores entre babysitter d'un côté et mannequin/ modèle de l'autre, visible sur la figure 7. Si babysitter est hautement stéréotypé féminin chez Misersky et al (2014) $(m=0.90)$, ce nom arrive à peine à un score neutre dans notre étude $(m=0.41)$. En revanche, mannequin et modèle, qui ont un score de stéréotypicalité légèrement plus bas que babysitter chez Misersky et al (2014) ( $m=0.83$ et 0.86 ), ont un score moyen indiquant bel et bien une interprétation non biaisée (mannequin, $m=0.57$; modèle, $m=0.56$ ). Autrement dit, l'étude de Misersky et al suggère que babysitter est plus fortement associé au stéréotype féminin que mannequin et modèle. Si les stéréotypes (féminins) étaient purement responsables de ces différences d'interprétation, nous nous attendrions à ce que babysitter ait un score plus élevé que mannequin et modèle. Or ce n'est pas ce que nous observons ici. Lévy, Gygax, \& Gabriel (2014) ont regardé les conditions favorisant l'activation de l'interprétation générique du masculin. Ces auteur·e's ont ainsi montré que la fréquence d'exposition d'un nom au masculin référant à une femme - qu'elles et ils nomment "influence implicite » - avait une influence plus forte sur l'activation de l'interprétation générique du masculin que de rappeler aux locuteurs et locutrices la règle de son emploi générique - «influence explicite »-. En d'autres termes, plus nous sommes exposés à des formes masculines désignant concrètement des femmes, moins l'analogie genre grammatical-genre social est faite. En ce qui concerne les résultats de notre étude, il est communément admis que modèle et mannequin sont utilisés au genre grammatical masculin et peuvent autant désigner des femmes que des hommes. Les moyennes obtenues pour ces noms vont dès lors dans le sens que de ce que les auteur·e's prédisaient et peuvent donc être attendus. Les figures 9 et 10 ci-dessous montrent les fréquences d'utilisation sur Google Ngram des genres grammaticaux masculin et féminin (marqués par le déterminant défini singulier) pour ces deux noms et illustrent la large préférence pour le genre masculin. Si l'on compare par exemple ces fréquences avec la proportion de le et la pour le nom journaliste (figure 11), le fait que le masculin est privilégié pour modèle et mannequin saute alors aux yeux. Si dans ces deux cas, le féminin est n'est presque jamais utilisé (les occurrences pour l'année 2008 sont quasiment à 0), la journaliste représente en revanche $0.0001 \%$ du total des tokens qui composent Google Ngram, contre $0.0005 \%$ pour le journaliste. Même si ces chiffres sont bas, et que le féminin est moins utilisé que le masculin, il est quand même plus fréquent que pour modèle et mannequin. 
Figure 9 : Proportion de le mannequin vs la mannequin sur Google Ngram Viewer

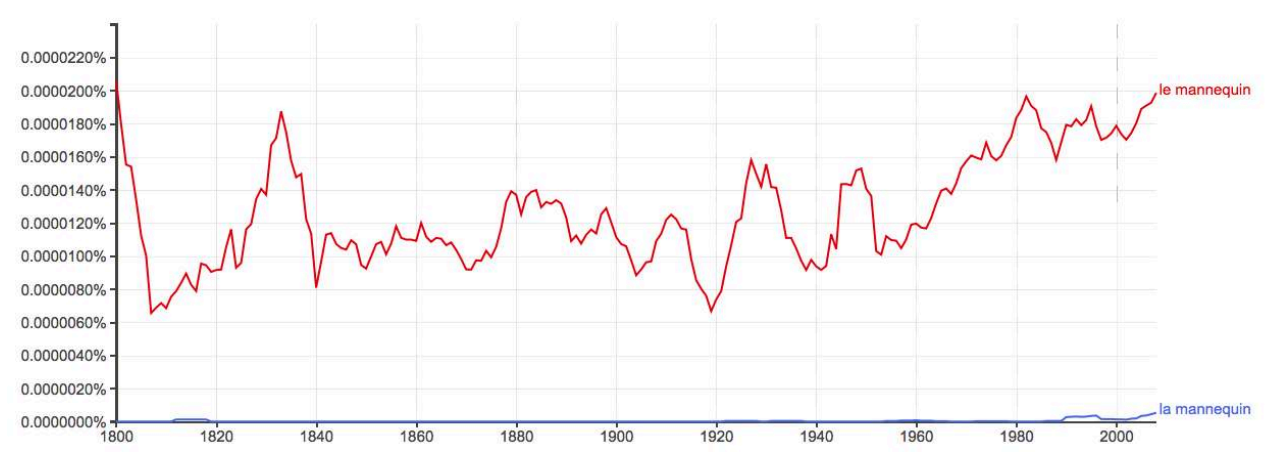

Figure 10 : Proportion de le modèle vs la modèle sur Google Ngram Viewer

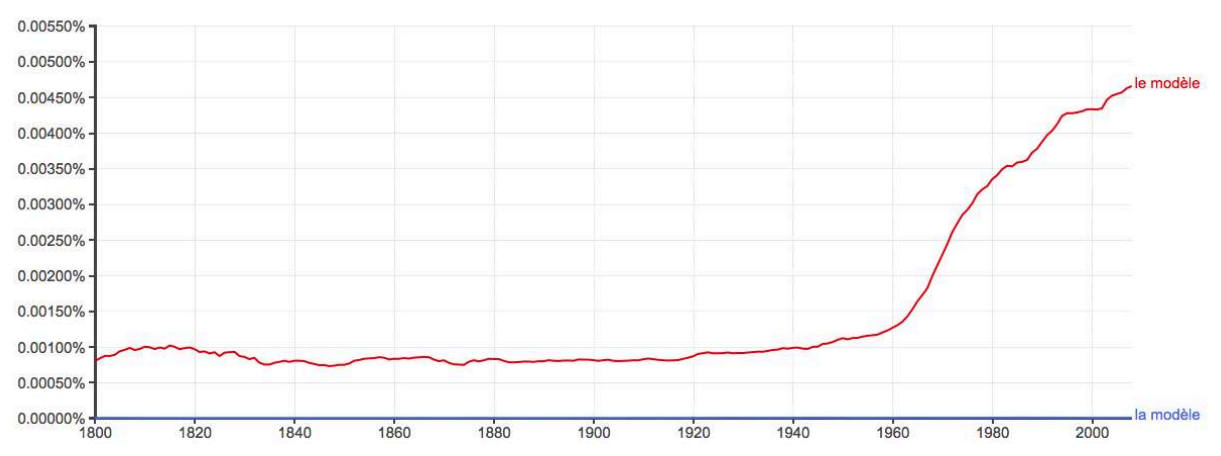

Figure 11 : Proportion de le journaliste vs la journaliste sur Google Ngram Viewer

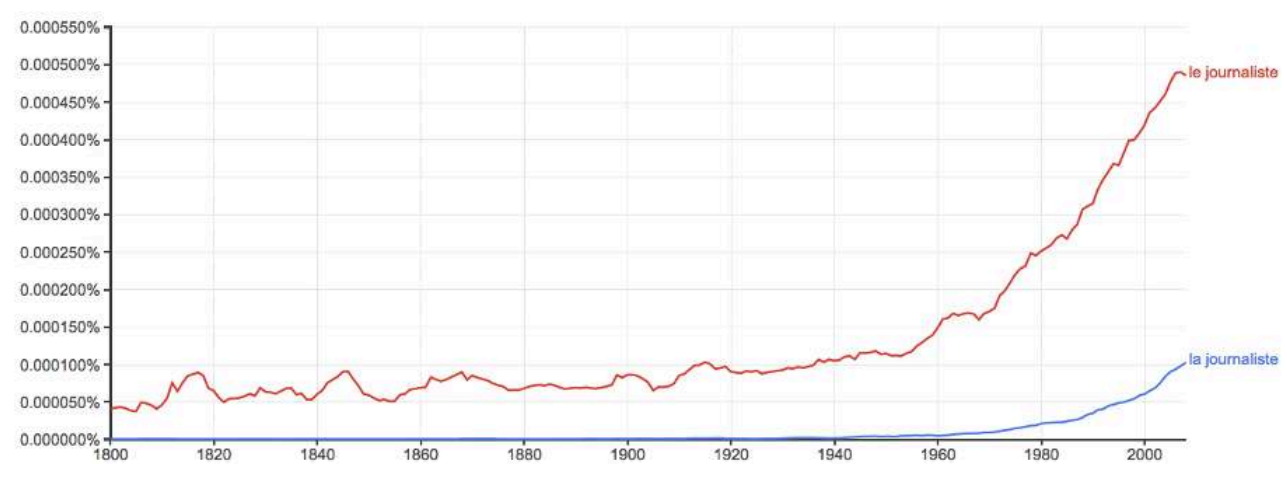

49 La proportion d'utilisation de ces lexèmes est cependant à prendre avec précaution. Premièrement, Google Ngram inclut tous les homographes donc toutes ces occurrences ne réfèrent pas exclusivement à des humains. Par ailleurs, l'utilisation des déterminants masculins (vs féminin) ajoute une nouvelle asymétrie dans les comptes. Le inclut aussi les hommes mannequins et modèles. Nous ne pouvons donc pas calculer la fréquence exacte des emplois du masculin et féminin pour référer seulement à des femmes. Néanmoins, la tendance reste que le masculin est préféré pour parler des mannequins et modèles femmes.

50 Afin de vérifier si les effets du genre grammatical et des stéréotypes sociaux observés sont réels, nous procédons à une analyse statistique de nos données. 


\section{Analyses statistiques}

51 Comme nous l'avons spécifié plus haut, nous observons la capacité du genre grammatical et des stéréotypes à influencer les locuteurs et locutrices dans leur choix pour déterminer le genre social d'un référent. Nous avons donc encore utilisé un modèle linéaire mixte sur R (lmer, package lme4, (Bates et al 2015)) pour prédire les scores d'interprétation des noms en fonction du genre grammatical et du stéréotype associé (variable continue correspondant aux scores Misersky et al (2014)). Les participantes et participants et les items sont utilisés comme facteurs aléatoires.

Tableau 2 : effets fixes du modèle linéaire. Intercept : genre grammatical masculin, âge moyen et participantes.

\begin{tabular}{|l|l|l|l|l|l|l|}
\hline & Estimate & Std. Error & $\mathrm{df}$ & $\mathrm{t}$ value & $\operatorname{Pr}(>|\mathrm{t}|)$ & \\
\hline (Intercept) & $1.969 \mathrm{e}-01$ & $3.431 \mathrm{e}-02$ & $1.168 \mathrm{e}+02$ & 5.739 & $7.65 \mathrm{e}-08$ & $* * *$ \\
\hline Genregram. féminin & $6.202 \mathrm{e}-01$ & $2.644 \mathrm{e}-02$ & $2.132 \mathrm{e}+03$ & 23.458 & $<2 \mathrm{e}-16$ & $* * *$ \\
\hline Scores Misersky (2014) & $2.700 \mathrm{e}-01$ & $4.152 \mathrm{e}-02$ & $8.548 \mathrm{e}+01$ & 6.503 & $5.07 \mathrm{e}-09$ & $* * *$ \\
\hline Genre. fem*scores & $-1.376 \mathrm{e}-01$ & $4.937 \mathrm{e}-02$ & $2.132 \mathrm{e}+03$ & -2.787 & 0.00537 & $* *$ \\
\hline+50 ans & $-1.924 \mathrm{e}-02$ & $3.402 \mathrm{e}-02$ & $5.600 \mathrm{e}+01$ & -0.566 & 0.57390 & \\
\hline $18-25$ ans & $-3.713 \mathrm{e}-02$ & $2.999 \mathrm{e}-02$ & $5.600 \mathrm{e}+01$ & -1.238 & 0.22089 & \\
\hline Hommes & $-2.265 \mathrm{e}-03$ & $2.484 \mathrm{e}-02$ & $5.600 \mathrm{e}+01$ & -0.091 & 0.92767 & \\
\hline Genre autre & $-4.077 \mathrm{e}-02$ & $5.852 \mathrm{e}-02$ & $5.600 \mathrm{e}+01$ & -0.698 & 0.48895 & \\
\hline
\end{tabular}

52 Notre modèle (tableau 2) indique un effet fixe du genre grammatical, c'est-à-dire que tant le féminin que le masculin ont globalement une influence sur la perception du genre (social) d'un référent. Nous constatons aussi un effet fixe des scores de stéréotypicalité. Ceci dit, il existe aussi un effet (négatif) de l'interaction entre ces scores et le genre grammatical: le genre grammatical a moins d'effet sur l'interprétation des noms avec des stéréotypes féminins que les noms à stéréotype masculin ou neutre. On peut facilement comprendre ce résultat dans le sens que si, sur la base du nom dans le syntagme (comme babysitter, secrétaire etc.), grâce au stéréotype, on est déjà presque certain·e que le référent est féminin, alors voir la babysitter ou la secrétaire ne va pas énormément augmenter sa croyance que le référent est une femme. Par contre, voir la diplomate ou la croque mort va significativement augmenter la croyance que le référent est une femme car le nom (diplomate, croque mort) suggère fortement le contraire. Vervecken et al. (2015) montraient déjà cette tendance sur les noms au pluriel. Pour les noms de métier portant un stéréotype féminin, bien que ces derniers soient marqués du masculin pluriel, les femmes étaient perçues comme pouvant mieux réussir que les hommes. A l'inverse, utiliser un syntagme inclusif (ex : les infirmiers et les infirmières) équilibrait les représentations des participantes et participants quant à la proportion de femmes et d'hommes pouvant réussir dans cette profession. L'effet du genre grammatical, tout comme l'effet des stéréotypes nus 
(section 3) est constant à travers les classes d'âges et le genre des participantes et participants, puisque notre modèle ne montre aucun effet significatif de l'âge ou du genre (social) sur l'interprétation des noms.

Les figures 12 et 13 ci-dessous montrent les régressions linéaires pour l'interprétation des noms selon le genre grammatical. La figure 12 illustre la prédiction pour l'interprétation du genre féminin, et la figure 13 pour le genre masculin. Nous constatons effectivement une pente différente pour ces deux genres, où les scores prédits pour le féminin vont environ de 0.8 pour les noms les plus stéréotypiquement masculins à 0.88 pour les noms les plus stéréotypiquement féminins. Les scores prédits pour le masculin s'étendent en revanche d'environ 0.25 pour les noms les plus masculins à 0.40 pour les stéréotypes les plus féminins.

Figure 12 : Régression linéaire des scores d'interprétation du genre féminin

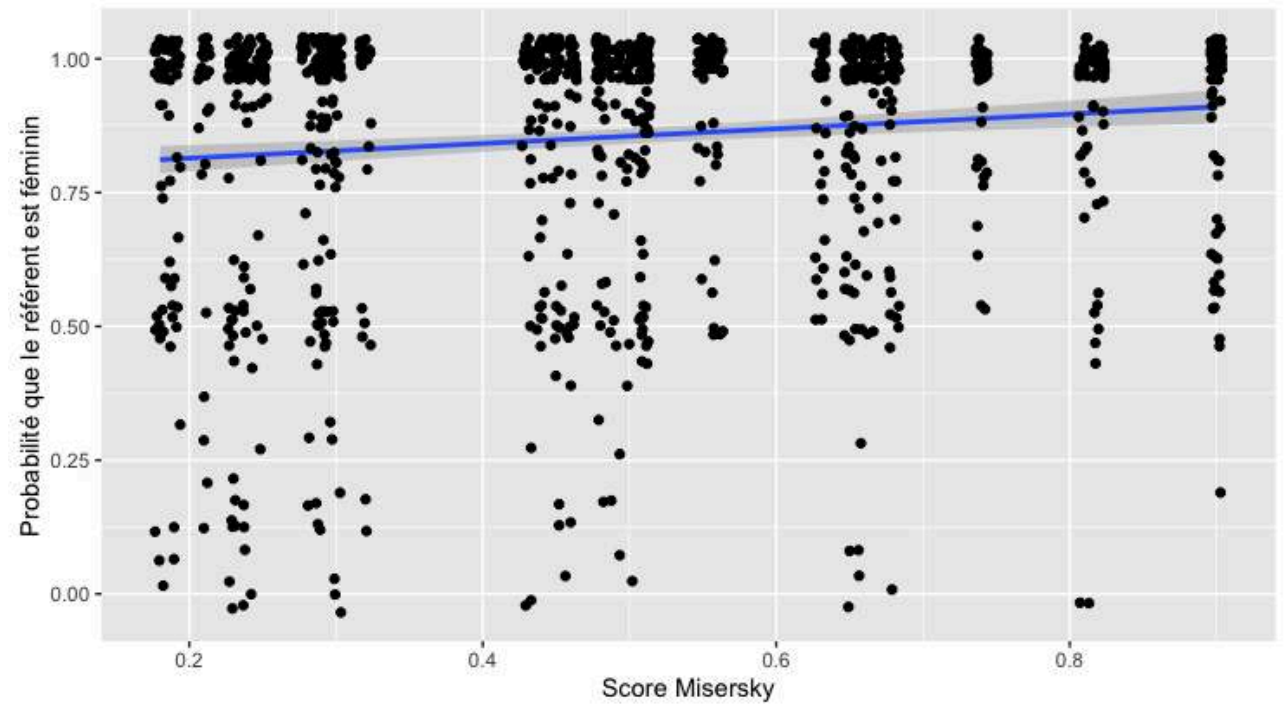

Figure 13 : Régression linéaire des scores d'interprétation du genre masculin

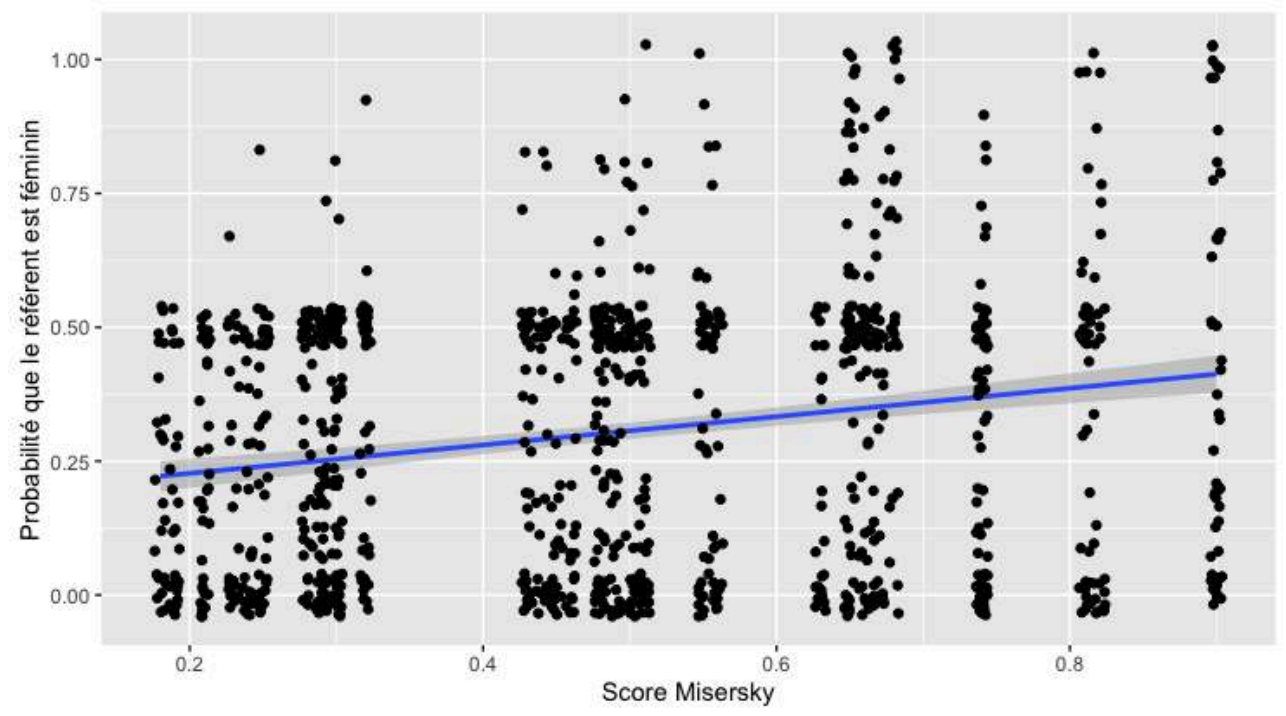


54 La figure 14 compare les lignes indiquant la relation entre le score Misersky et la moyenne de l'interprétation des syntagmes nominaux neutralisés (en vert), féminins (en rouge) et masculins (en bleu). $\mathrm{Si}$, comme soutient la tradition grammaticale française, le genre grammatical masculin n'ajoute aucun sens «masculin » aux syntagmes, son interprétation devrait être équivalente à l'interprétation des syntagmes où le genre est neutralisé. C'est à dire, la ligne verte et la ligne bleue devraient se chevaucher partout dans le graphique. Or, cette tendance se retrouve seulement pour les noms à stéréotype masculin: les lignes bleues et vertes sont très proches. Par ailleurs, la ligne verte n'atteint jamais la ligne rouge, alors qu'elle dépasse même la ligne bleue. Néanmoins, les t.test sur les différences de moyennes indiquent que les noms marqués tant au masculin qu'au féminin ont des moyennes significativement différentes des moyennes obtenues lorsque le genre est neutralisé $(p<.0001)$.

Figure 14 : Comparaison des jugements d'interprétation des syntagmes marqués féminins, masculins ou neutralisés selon le score Misersky du nom.

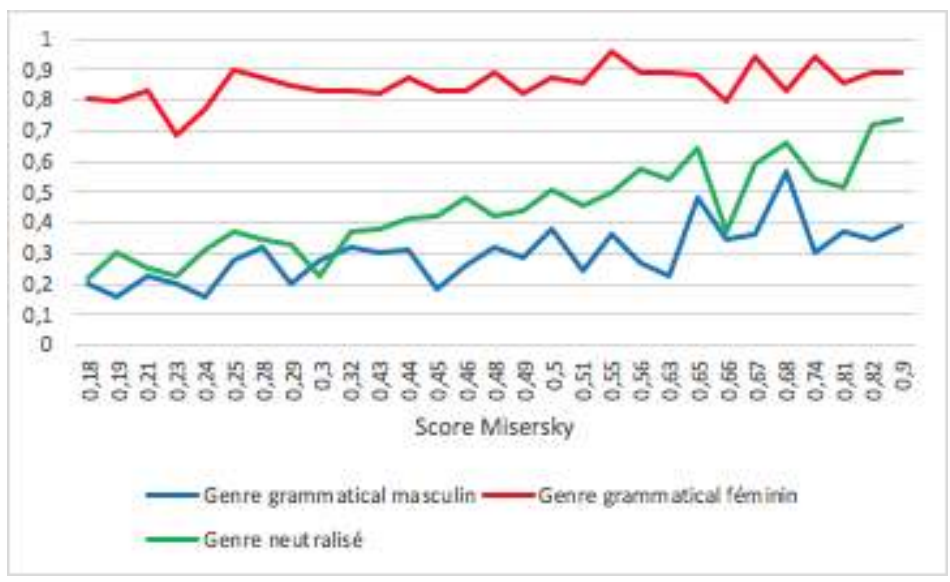

\section{Discussion générale}

Nos données permettent de voir l'effet dont parlait Cameron pour l'anglais : chez les noms à stéréotype masculin (comme astronaut en anglais, ou diplomate, clown, croquemort, etc. en français), la forme non marquée par le genre crée une interprétation genrée similaire à la forme formellement marquée masculine. Cependant, quand on regarde les noms à stéréotype féminin deux éléments apparaissent. D'une part, il existe souvent un écart entre les croyances des participantes et participants après la lecture d'un syntagme neutralisé et un syntagme marqué masculin. D'autre part, les croyances en fonction de la neutralisation du genre et du marquage féminin sont différentes. Autrement dit, nous avons regardé si (1) les noms à stéréotype masculin ont des moyennes différentes avec genre masculin et avec le genre neutralisé et (2) si les moyennes des noms à stéréotype féminin diffèrent selon le marquage au féminin ou la neutralisation.

Nos résultats ont au moins deux conséquences politiques importantes. Tout d'abord, bien que le genre féminin soit moins informatif lorsqu'il marque un nom à fort stéréotype féminin, la différence entre les lignes rouges et vertes en figure 14 montre l'importance d'utilisation d'un marquage explicite pour déclencher une représentation mentale féminine du référent. Ensuite, la différence entre les lignes bleues et vertes 
indique que quand les locutrices et locuteurs utilisent un syntagme marqué masculin, cela crée une représentation qui est, dans presque tous les cas, plus masculine que la réalité. Comme le montre la figure 15 , les scores d'interprétation ne reflètent pas du tout les proportions données par l'INSEE.

Figure 15 : Scores d'interprétation (genre grammatical masculin) vs proportions de femmes dans ces métiers selon l'INSEE.

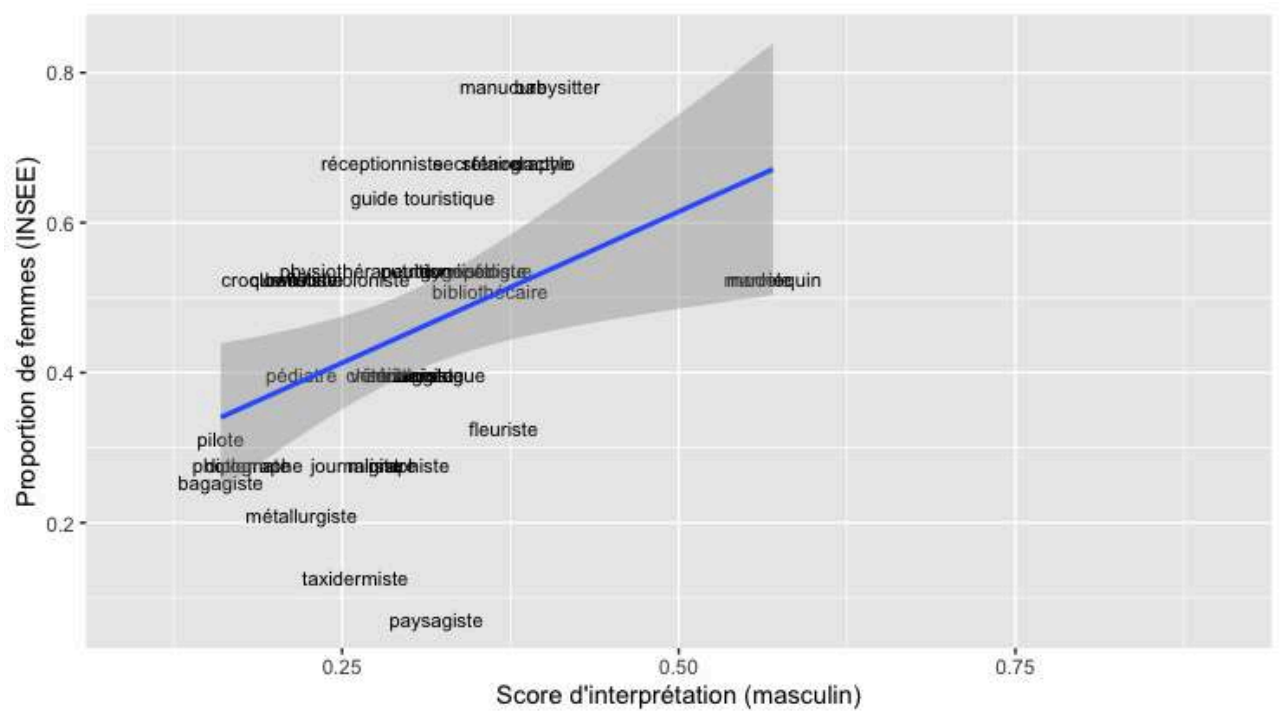

57 Par conséquent, nos résultats renforcent ceux discutés par la (récente) recherche en psycholinguistique dans la mesure où nous arrivons aux mêmes conclusions : le genre grammatical masculin crée un réel biais masculin, un biais qui va au-delà des stéréotypes nominaux. Les stéréotypes en revanche, a minima concernant les noms étudiés ici, se rapprochent des distributions que l'on retrouve dans la société française.

\section{Conclusion}

Dans cet article, nous avons contribué au développement d'une littérature psycholinguistique croissante sur le traitement et l'interprétation du genre grammatical en français. Nous avons présenté deux expériences qui visaient à mesurer l'effet du genre grammatical et des stéréotypes de noms de métiers sur les représentations mentales des locuteurs et locutrices francophones. En comparant les syntagmes nominaux où le genre grammatical était marqué avec ceux où le genre était neutralisé, nous avons pu démêler la contribution des stéréotypes du marquage formel du genre, quelque chose qui n'avait pas encore été fait pour le français.

Nos deux études ont produit des résultats similaires aux études précédentes en psycholinguistique : comme Gygax et al. (2008) ont montré pour le pluriel, et contre la tradition grammaticale française, nous avons trouvé que le genre grammatical joue un rôle très important dans l'interprétation genrée des syntagmes nominaux en français. Comme Garnham et al. (2012), nous avons également trouvé que les stéréotypes associés avec les noms de métier jouent aussi un rôle dans la représentation du genre du référent.

Pour finir, nos expériences n'indiquent aucun effet de l'âge sur l'interprétation du genre du référent. Mais comme notre design a très peu contrôlé les facteurs sociaux 
(âge, niveau d'éducation et idéologies du genre (Parks \& Roberton 2004 ; Swim, Mallett \& Stangor 2004 ; Sarrasin, Gabriel, \& Gygax 2012)), nous ne sommes en réalité pas en mesure de discuter avec certitude des (non- ) effets de ces facteurs. L'étude de Burnett $\&$ Richy (en préparation) réplique en partie cette étude et discute des facteurs sociaux.

\section{BIBLIOGRAPHIE}

BAIDER, Fabienne. H., KHAZNADAR, Edwige \& MOREAU, Thérèse. 2007. « Les enjeux de la parité linguistique ». Nouvelles questions féministes, 26(3) : 4-12.

BATES, Douglas, MAECHLER, Martin, BOLKER, Ben, WALKER, Steve. 2015. « Fitting Linear MixedEffects Models Using lme4 ». Journal of Statistical Software, 67(1): 1-48

BEM, Sandra Lipsitz. 1993. The lenses of gender: transforming the debate on sexual inequality. New Haven : Yale University Press.

BRAUER, Markus \& LANDRY, Michaël. 2008. « Un ministre peut-il tomber enceinte ? L'impact du générique masculin sur les représentations mentales ». L'Année psychologique, 108(2) : 243-272.

BODINE, Ann. « Androcentrism in prescriptive grammar: Singular'they', sex-indefinite 'he', and 'he or she' ». Language in society, 1975: 129-146.

BOYCE, Veronica, VON DER MALSBURG, Titus, POPPELS, Tills \& LEVY, Roger. 2019. “Female gender is consistently under-expressed in pronoun production and under-inferred in comprehension". In the 93th Annual Meeting of the Linguistics Society of America. New York, PANCHEVA, Roumyana \& ISKAROUS, Khalil (eds), New York, USA : Linguistic Society of America CAMERON, Deborah. 1985. Feminism and Linguistic Theory, Basingstoke and London: MacMillan. CAMERON, Deborah. 2018. "The illusion of inclusion”. Language: a feminist guide Consulté le 01 avril 2020. URL : https://debuk.wordpress.com/2018/08/05/the-illusion-ofinclusion/

CARREIRAS, Manuel, GARNHAM, Alan, OAKHILL, Jane \& CAIN, Kate. 1996. " The use of stereotypical gender information in constructing a mental model: Evidence from English and Spanish ». Quarterly Journal of Experimental Psychology Section A-Human Experimental Psychology, 49(3): 639-663.

CHATARD, Armand, GUIMONT, Serge \& MARTINOT, Delphine. 2005. « Impact de la féminisation lexicale des professions sur l'auto-efficacité des élèves : une remise en cause de l'universalisme masculin? »'année psychologique, 105(2) : 249-272

CORBETT, Greville G. 1991. Gender. Cambridge ; New York : Cambridge university press.

ECKERT, Penelope. 1998. “Age as a Sociolinguistic Variable”. in The Handbook of Sociolinguistics, COULMAS, Florian (ed.), Cambridge: Blackwell, pp. 168-186

GABRIEL, Ute, GYGAX, Pascal, SARRASIN, Oriane, GARNHAM Alan \& OAKHILL, Jane. 2008. « Au pairs are rarely male : Norms on the gender perception of role names across English, French, and German ». Behavior Research Methods, 40(1): 206-212. 
GARNHAM, Alan, OAKHILL, Jane \& REYNOLDS, David. 2002. « Are inferences from stereotyped role names to characters' gender made elaboratively? » Memory \& Cognition, 30(3): 439-446.

GARNHAM, Alan, GABRIEL, Ute, SARRASIN, Oriane, GYGAX, Pascal \& OAKHILL, Jane. 2012. «Gender Representation in Different Languages and Grammatical Marking on Pronouns: When Beauticians, Musicians, and Mechanics Remain Men ». Discourse Processes, 49(6): 481-500.

GARNHAM, Alan, DOEHREN, Sam \& GYGAX, Pascal. 2015. “True gender ratios and stereotype rating norms". Frontiers in psychology, $6: 1023$.

GREVISSE, Maurice \& GOOSSE, André. 2008. Le bon usage : grammaire française (14e édition). Paris : Duculot.

GYGAX, Pascal \& GABRIEL, Ute. 2008. « Can a group of musicians be composed of women? Generic interpretation of French masculine role names in the absence and presence of feminine forms ». Swiss Journal of Psychology, 67(3): 143-151.

GYGAX, Pascal, GABRIEL, Ute, SARRASIN, Oriane, OAKHILL, Jane \& GARNHAM, Alan. 2008. « Generically intended, but specifically interpreted: When beauticians, musicians, and mechanics are all men ». Language and Cognitive Processes, 23(3): 464-485.

GYGAX, Pascal, GABRIEL, Ute, LÉVY, Ariane, POOL, Eva, GRIVEL, Marjorie \& Pedrazzini, Elena. 2012. « The masculine form and its competing interpretations in French: When linking grammatically masculine role names to female referents is difficult ». Journal of Cognitive Psychology, 24(4): 395-408.

GYGAX, Pascal, GARNHAM, Alan \& DOEHREN, Sam. 2016. "What do true gender ratios and stereotype norms really tell us?” Frontiers in psychology, 7: 1036

GYGAX, Pascal, GABRIEL, Ute \& ZUFFEREY, Sandrine. 2019. « Le masculin et ses multiples sens : Un problème pour notre cerveau... et notre société ». Savoirs en Prisme, 10.

GYGAX, Pascal, SCHOENHALS, Lucie, LÉVY, Arik, LUETHOLD, Patrick \& GABRIEL, Ute. 2019. "Exploring the onset of a male-biased interpretation of masculine generics among French speaking kindergarten children". Frontiers in psychology, 10: 1225

HOCKETT, Charles. 1958. A course in modern linguistics. New York : The Macmillan company. HOUDEBINE-GRAVAUD, Anne-Marie. 1995. « Des femmes dans la langue et les discours ». Annales de Normandie, 26(1) : 385-398.

IRMEN, Lisa \& KUROVSKAJA, Julia. 2010. « On the semantic content of grammatical gender and its impact on the representation of human referents ». Experimental Psychology, 57(5): 367-375.

JAKOBSON, Roman. 1939. « Signe Zéro », dans Jakobson, Roman (ed.). Selected writings II. The Hague \& Paris : Mouton, $1971: 211-219$.

KHAZNADAR, Edwidge. 2007. « Le non-genre académique : doctrine de la domination masculine en France ». Nouvelles Questions Féministes, 26(3) : 25-38.

LABOV, William. 2000. Principles of linguistic change: Volume 2 : social factors. Oxford : Blackwell. LÉVY, Ariane, GYGAX, Pascal \& GABRIEL, Ute. 2014. « Fostering the generic interpretation of grammatically masculine forms: When my aunt could be one of the mechanics ». Journal of Cognitive Psychology, 26(1): 27-38.

MATELL, Michael S. \& JACOBY, Jacob. 1972. "Is there an optimal number of alternatives for Likert scale items? Study I: Reliability and validity”. Educational and psychological measurement, 31(3): 657-674. 
MARTINET, André. 1999. « Genre et sexe ». La linguistique, 35(2) : 5-9.

MICHARD, Claire. 1996. «Genre et sexe en linguistique : les analyses du masculin générique. » Mots. Les langages du politique, 49(1): 29-47

MICHARD, Claire. 1999. « Humain / femelle : deux poids deux mesures dans la catégorisation de sexe en français ». Nouvelles Questions Féministes, 20(1): 53-95.

MICHARD, Claire. 2001. Le sexe en linguistique : sémantique ou zoologie? Paris : L'Harmattan MICHARD, Claire. 2019. Humain / femelle de l'humain : effet idéologique du rapport de sexage et de notion de sexe en français. Les éditions sans fin.

MICHEL, Lucy. 2016. La relation entre genre grammatical et dénomination de la personne en langue française. Approches sémantiques. Thèse de doctorat. Université de Bourgogne.

MISERSKY, Julie, GYGAX, Pascal., CANAL, Paolo., GABRIEL, U. 2014. « Norms on the gender perception of role nouns in Czech, English, French, German, Italian, Norwegian, and Slovak ». Behavior Research Methods, 46(3): 841-871.

PARKS, Janet. B. \& ROBERTON, Maryanne. 2004. “Attitudes toward women mediate the gender effect on attitudes toward sexist language”. Psychology of Women Quarterly, 28: 233- 239.

R CORE TEAM. 2020. R: A language and environment for statistical computing. R Foundation for Statistical Computing, Vienna, Austria. URL https://www.R-project.org/

SARRASIN, Oriane, GABRIEL, Ute \& GYGAX, Pascal. 2012. « Sexism and Attitudes Toward GenderNeutral Language: The Case of English, French, and German ». Swiss Journal of Psychology, 71(3): $113-124$

SCHAFROTH, Elmar. 2003. "Gender in French. Structural properties, incongruences and asymmetries". In Gender across languages: The linguistic representation of women and men. (Vol. 3), HELLINGER, Marlis, \& MOTSCHENBACHER, Heiko. (Eds.). Amsterdam, Philadelphia: John Benjamins Publishing Company, pp. 87-117.

SPENDER, Dale. 1985. Man made language. London, GB: Routledge and Regan Paul SWIM, Janet. K., MALLETT, Robyn \& STANGOR, Charles. 2004. "Understanding subtle sexism: Detection and use of sexist language". Sex Roles, 51: 117-128.

VERVECKEN, Dries, GYGAX, Pascal, GABRIEL, Ute, GUILLOD, Matthias, \& HANNOVER, Bettina. 2015. "Warm-hearted businessmen, competitive housewives? Effects of gender-fair language on adolescents' perceptions of occupations". Frontiers in psychology, 6: 1437

\section{ANNEXES}

\section{Annexe 1 : stimuli}

\begin{tabular}{|l|l|l|}
\hline Item & Adjectifs & Stimuli \\
\hline $\begin{array}{l}\text { Item } \\
1\end{array}$ & $\begin{array}{l}\text { incorruptible, } \\
\text { imperturbable, } \\
\text { indispensable }\end{array}$ & $\begin{array}{l}\text { Pour téléphoner, le/la diplomate/secrétaire/journaliste appelle } \\
\text { toujours en numéro masqué. }\end{array}$ \\
\hline
\end{tabular}




\begin{tabular}{|c|c|c|}
\hline $\begin{array}{l}\text { Item } \\
2\end{array}$ & $\begin{array}{l}\text { imperturbable, } \\
\text { indispensable, } \\
\text { incorruptible }\end{array}$ & $\begin{array}{l}\text { Quand son travail est fini, le/la secrétaire/journaliste/diplomate } \\
\text { fait toujours une sauvegarde. }\end{array}$ \\
\hline $\begin{array}{l}\text { Item } \\
3\end{array}$ & $\begin{array}{l}\text { indispensable, } \\
\text { Incorruptible, } \\
\text { imperturbable }\end{array}$ & $\begin{array}{l}\text { Si l'ordinateur est cassé, le/la journaliste/diplomate/secrétaire } \\
\text { note tout dans son agenda. }\end{array}$ \\
\hline $\begin{array}{l}\text { Item } \\
4\end{array}$ & $\begin{array}{l}\text { Efficace, } \\
\text { Aimable, } \\
\text { insondable }\end{array}$ & $\begin{array}{l}\text { Pendant les vacances d'hiver, la/le ministre/réceptionniste/ } \\
\text { graphiste part au ski. }\end{array}$ \\
\hline $\begin{array}{l}\text { Item } \\
5\end{array}$ & $\begin{array}{l}\text { Aimable, } \\
\text { Insondable, } \\
\text { efficace }\end{array}$ & $\begin{array}{l}\text { Pour l'heure du déjeuner, la/le réceptionniste/graphiste/ministre } \\
\text { mange un sandwich. }\end{array}$ \\
\hline $\begin{array}{l}\text { Item } \\
6\end{array}$ & $\begin{array}{l}\text { Insondable, } \\
\text { Efficace, } \\
\text { aimable }\end{array}$ & $\begin{array}{l}\text { Quand il pleut, la/le graphiste/ministre/réceptionniste sort avec } \\
\text { son parapluie. }\end{array}$ \\
\hline $\begin{array}{l}\text { Item } \\
7\end{array}$ & $\begin{array}{l}\text { Unique, } \\
\text { Admirable, } \\
\text { inaccessible }\end{array}$ & $\begin{array}{l}\text { La veille d'un spectacle, le/la bassiste/flutiste/violoniste a } \\
\text { toujours le trac. }\end{array}$ \\
\hline $\begin{array}{l}\text { Item } \\
8\end{array}$ & $\begin{array}{l}\text { Admirable, } \\
\text { Inaccessible, } \\
\text { unique }\end{array}$ & Le/la flutiste/violoniste/bassiste fait de sa passion son métier \\
\hline $\begin{array}{l}\text { Item } \\
9\end{array}$ & $\begin{array}{l}\text { Inaccessible, } \\
\text { Unique, } \\
\text { admirable }\end{array}$ & $\begin{array}{l}\text { Pour ses jours de congés, le violoniste/bassiste/flutiste aime aller } \\
\text { à la mer. }\end{array}$ \\
\hline $\begin{array}{l}\text { Item } \\
10\end{array}$ & $\begin{array}{l}\text { Empathique, } \\
\text { Incontournable, } \\
\text { acceptable }\end{array}$ & $\begin{array}{l}\text { Avant ses rendez-vous, la/le neurologue/nutritionniste/ } \\
\text { gynécologue lit des revues scientifiques. }\end{array}$ \\
\hline $\begin{array}{l}\text { Item } \\
11\end{array}$ & $\begin{array}{l}\text { Incontournable, } \\
\text { Acceptable, } \\
\text { empathique }\end{array}$ & $\begin{array}{l}\text { Quand il y a grève, la/le nutritionniste/gynécologue/neurologue } \\
\text { va au travail en vélo. }\end{array}$ \\
\hline
\end{tabular}




\begin{tabular}{|c|c|c|}
\hline $\begin{array}{l}\text { Item } \\
12\end{array}$ & $\begin{array}{l}\text { Acceptable, } \\
\text { Empathique, } \\
\text { incontournable }\end{array}$ & $\begin{array}{l}\text { Après chaque visite, la/le gynécologue/neurologue/nutritionniste } \\
\text { se lave les mains. }\end{array}$ \\
\hline $\begin{array}{l}\text { Item } \\
13\end{array}$ & $\begin{array}{l}\text { Intrépide, } \\
\text { Incollable, } \\
\text { idéaliste }\end{array}$ & $\begin{array}{l}\text { Ayant souvent affaire à des gens pénibles, le/la bagagiste/ } \\
\text { manucure/guide touristique se doit de rester calme. }\end{array}$ \\
\hline $\begin{array}{l}\text { Item } \\
14\end{array}$ & $\begin{array}{l}\text { Incollable, } \\
\text { Idéaliste, } \\
\text { intrépide }\end{array}$ & $\begin{array}{l}\text { Pour partir en voyage, le/la manucure/guide touristique/ } \\
\text { bagagiste préfère prendre le train. }\end{array}$ \\
\hline $\begin{array}{l}\text { Item } \\
15\end{array}$ & $\begin{array}{l}\text { Idéaliste, } \\
\text { Intrépide, } \\
\text { incollable }\end{array}$ & $\begin{array}{l}\text { Le vendredi soir, le/la guide touristique/bagagiste/manucure aime } \\
\text { aller au cinéma. }\end{array}$ \\
\hline $\begin{array}{l}\text { Item } \\
16\end{array}$ & $\begin{array}{l}\text { Habile, } \\
\text { Inqualifiable, } \\
\text { émérite }\end{array}$ & $\begin{array}{l}\text { Quand il fait beau, la/le paysagiste/fleuriste/zoologiste fait des } \\
\text { balades en forêt. }\end{array}$ \\
\hline $\begin{array}{l}\text { Item } \\
17\end{array}$ & $\begin{array}{l}\text { Inqualifiable, } \\
\text { Émérite, } \\
\text { habile }\end{array}$ & $\begin{array}{l}\text { Le dimanche des élections, la/le fleuriste/zoologiste/paysagiste se } \\
\text { rend dans son bureau de vote. }\end{array}$ \\
\hline $\begin{array}{l}\text { Item } \\
18\end{array}$ & $\begin{array}{l}\text { Émérite, } \\
\text { Habile, } \\
\text { inqualifiable }\end{array}$ & $\begin{array}{l}\text { Selon les saisons, la/le zoologiste/paysagiste/fleuriste a des tâches } \\
\text { différentes à effectuer. }\end{array}$ \\
\hline $\begin{array}{l}\text { Item } \\
19\end{array}$ & $\begin{array}{l}\text { Atypique, } \\
\text { Indescriptible, } \\
\text { énigmatique }\end{array}$ & $\begin{array}{l}\text { Avec son métier, le/la clown/mannequin/photographe est souvent } \\
\text { en déplacement. }\end{array}$ \\
\hline $\begin{array}{l}\text { Item } \\
20\end{array}$ & $\begin{array}{l}\text { Indescriptible, } \\
\text { Énigmatique, } \\
\text { atypique }\end{array}$ & $\begin{array}{l}\text { Tous les ans le/la mannequin/photographe/clown fête le nouvel } \\
\text { an en Espagne. }\end{array}$ \\
\hline $\begin{array}{l}\text { Item } \\
21\end{array}$ & $\begin{array}{l}\text { Énigmatique, } \\
\text { Atypique, } \\
\text { indescriptible }\end{array}$ & $\begin{array}{l}\text { Pour se maintenir en forme, le/la photographe/clown/mannequin } \\
\text { va à la piscine. }\end{array}$ \\
\hline
\end{tabular}




\begin{tabular}{|c|c|c|}
\hline $\begin{array}{l}\text { Item } \\
22\end{array}$ & $\begin{array}{l}\text { Incorrigible, } \\
\text { Affable, } \\
\text { éclectique }\end{array}$ & $\begin{array}{l}\text { Tous les matins, la/le chimiste/logopédiste/biologiste boit un café } \\
\text { pour se réveiller. }\end{array}$ \\
\hline $\begin{array}{l}\text { Item } \\
23\end{array}$ & $\begin{array}{l}\text { Affable, } \\
\text { Éclectique, } \\
\text { incorrigible }\end{array}$ & $\begin{array}{l}\text { Plusieurs fois dans l'année, la/le logopédiste/biologiste/chimiste } \\
\text { assiste à des conférences. }\end{array}$ \\
\hline $\begin{array}{l}\text { Item } \\
24\end{array}$ & $\begin{array}{l}\text { Éclectique, } \\
\text { Incorrigible, } \\
\text { affable }\end{array}$ & $\begin{array}{l}\text { Pendant le week-end, la/le biologiste/chimiste/logopédiste se } \\
\text { repose. }\end{array}$ \\
\hline $\begin{array}{l}\text { Item } \\
25\end{array}$ & $\begin{array}{l}\text { intègre, } \\
\text { Inlassable, } \\
\text { inébranlable }\end{array}$ & $\begin{array}{l}\text { Le/la croque-mort/modèle/physiothérapeute achète le journal au } \\
\text { kiosque. }\end{array}$ \\
\hline $\begin{array}{l}\text { Item } \\
26\end{array}$ & $\begin{array}{l}\text { Inlassable, } \\
\text { Inébranlable, } \\
\text { intègre }\end{array}$ & $\begin{array}{l}\text { Afin de garder bonne mine l'hiver, le/la modèle/ } \\
\text { physiothérapeute/croque-mort fait des U.V. }\end{array}$ \\
\hline $\begin{array}{l}\text { Item } \\
27\end{array}$ & $\begin{array}{l}\text { Inébranlable, } \\
\text { Intègre, } \\
\text { inlassable }\end{array}$ & $\begin{array}{l}\text { Quand il fait froid, le/la physiothérapeute/croque-mort/modèle } \\
\text { met un col roulé. }\end{array}$ \\
\hline $\begin{array}{l}\text { Item } \\
28\end{array}$ & $\begin{array}{l}\text { Enviable, } \\
\text { Irréprochable, } \\
\text { impeccable }\end{array}$ & $\begin{array}{l}\text { Après sa journée de travail, la/le taxidermiste/dactylo/ } \\
\text { sténographe range son bureau. }\end{array}$ \\
\hline $\begin{array}{l}\text { Item } \\
29\end{array}$ & $\begin{array}{l}\text { Irréprochable, } \\
\text { Impeccable, } \\
\text { enviable }\end{array}$ & $\begin{array}{l}\text { Pendant la canicule, la/le dactylo/sténographe/taxidermiste reste } \\
\text { à l'intérieur. }\end{array}$ \\
\hline $\begin{array}{l}\text { Item } \\
30\end{array}$ & $\begin{array}{l}\text { Impeccable, } \\
\text { Enviable, } \\
\text { irréprochable }\end{array}$ & $\begin{array}{l}\text { Les soirs de pleine lune, la/le sténographe/taxidermiste/dactylo } \\
\text { dort mal. }\end{array}$ \\
\hline $\begin{array}{l}\text { Item } \\
31\end{array}$ & $\begin{array}{l}\text { Irremplaçable, } \\
\text { Optimiste, } \\
\text { incroyable }\end{array}$ & $\begin{array}{l}\text { Pendant les vacances scolaires, le/la pilote/babysitter/pédiatre } \\
\text { travaille beaucoup. }\end{array}$ \\
\hline
\end{tabular}




\begin{tabular}{|l|l|l|}
\hline $\begin{array}{l}\text { Item } \\
32\end{array}$ & $\begin{array}{l}\text { optimiste, } \\
\text { Incroyable, } \\
\text { irremplaçable }\end{array}$ & A la fin du mois, le/la babysitter/pédiatre/pilote paie ses factures. \\
\hline $\begin{array}{l}\text { Item } \\
33\end{array}$ & $\begin{array}{l}\text { Incroyable, } \\
\text { Irremplaçable, } \\
\text { optimiste }\end{array}$ & $\begin{array}{l}\text { A chaque premier mai, le/la pédiatre/pilote/babysitter achète un } \\
\text { brin de muguet. }\end{array}$ \\
\hline $\begin{array}{l}\text { Item } \\
34\end{array}$ & $\begin{array}{l}\text { Agile, } \\
\text { Enthousiaste, }\end{array}$ & $\begin{array}{l}\text { Depuis la classe de CP, la/le métallurgiste/bibliothécaire/ } \\
\text { vétérinaire adore lire. }\end{array}$ \\
\hline $\begin{array}{l}\text { Item } \\
35\end{array}$ & $\begin{array}{l}\text { Immense } \\
\text { Enthousiaste, }\end{array}$ & $\begin{array}{l}\text { Tôt le matin, la/le bibliothécaire/vétérinaire/métallurgiste } \\
\text { aromène son chien. }\end{array}$ \\
\hline $\begin{array}{l}\text { Item } \\
36\end{array}$ & $\begin{array}{l}\text { Imile } \\
\text { Agile, }\end{array}$ & $\begin{array}{l}\text { Quand il y a les jeux olympiques, la/le vétérinaire/métallurgiste/ } \\
\text { bibliothécaire regarde la télévision. }\end{array}$ \\
\hline
\end{tabular}

Annexe 2 : scores moyens en interprétation

\begin{tabular}{|l|l|l|l|l|}
\hline ITEMS & $\begin{array}{l}\text { Score Miserky } \\
(2014)\end{array}$ & $\begin{array}{l}\text { Genre gram } \\
\text { masculin }\end{array}$ & $\begin{array}{l}\text { Genre } \\
\text { féminin }\end{array}$ & $\begin{array}{l}\text { Neutralisation du } \\
\text { genre }\end{array}$ \\
\hline Clown & 0,18 & 0,20 & 0,81 & 0,22 \\
\hline Bagagiste & 0,19 & 0,16 & 0,80 & 0,30 \\
\hline Métallurgiste & 0,21 & 0,23 & 0,83 & 0,25 \\
\hline Croque-mort & 0,23 & 0,20 & 0,69 & 0,23 \\
\hline Pilote & 0,24 & 0,16 & 0,77 & 0,31 \\
\hline Ministre & 0,25 & 0,28 & 0,90 & 0,37 \\
\hline Paysagiste & 0,28 & 0,32 & 0,87 & 0,35 \\
\hline Diplomate & 0,29 & 0,18 & 0,85 & 0,32 \\
\hline Bassiste & 0,29 & 0,22 & 0,84 & 0,34 \\
\hline Taxidermiste & 0,30 & 0,26 & 0,76 & 0,23 \\
\hline Chimiste & 0,30 & 0,28 & 0,90 & 0,35 \\
\hline
\end{tabular}




\begin{tabular}{|c|c|c|c|c|}
\hline Neurologue & 0,32 & 0,32 & 0,83 & 0,37 \\
\hline Graphiste & 0,43 & 0,30 & 0,82 & 0,38 \\
\hline Guide touristique & 0,44 & 0,31 & 0,87 & 0,41 \\
\hline Photographe & 0,45 & 0,18 & 0,83 & 0,42 \\
\hline Journaliste & 0,46 & 0,26 & 0,83 & 0,48 \\
\hline Zoologiste & 0,48 & 0,33 & 0,88 & 0,45 \\
\hline Biologiste & 0,48 & 0,31 & 0,90 & 0,39 \\
\hline Vétérinaire & 0,49 & 0,29 & 0,82 & 0,44 \\
\hline Sténographe & 0,50 & 0,38 & 0,87 & 0,51 \\
\hline Physiothérapeute & 0,51 & 0,26 & 0,86 & 0,46 \\
\hline Pédiatre & 0,51 & 0,22 & 0,86 & 0,45 \\
\hline Gynécologue & 0,55 & 0,36 & 0,96 & 0,50 \\
\hline Violoniste & 0,56 & 0,27 & 0,89 & 0,58 \\
\hline Flutiste & 0,63 & 0,23 & 0,89 & 0,54 \\
\hline Dactylo & 0,65 & 0,40 & 0,89 & 0,64 \\
\hline Modèle & 0,65 & 0,56 & 0,86 & 0,63 \\
\hline Logopédiste & 0,66 & 0,35 & 0,80 & 0,37 \\
\hline Bibliothécaire & 0,67 & 0,36 & 0,94 & 0,59 \\
\hline Mannequin & 0,68 & 0,57 & 0,83 & 0,66 \\
\hline Réceptionniste & 0,74 & 0,28 & 0,95 & 0,54 \\
\hline Nutritionniste & 0,74 & 0,33 & 0,93 & 0,63 \\
\hline Fleuriste & 0,81 & 0,37 & 0,86 & 0,52 \\
\hline Secrétaire & 0,82 & 0,35 & 0,89 & 0,72 \\
\hline Babysitter & 0,90 & 0,41 & 0,89 & 0,73 \\
\hline Manucure & 0,90 & 0,37 & 0,89 & 0,75 \\
\hline
\end{tabular}




\section{NOTES}

1. Nous pouvons cependant noter le changement d'attitude de l'Académie Française face à la féminisation des noms de métiers et fonctions (voir leur rapport de 2019: La féminisation des noms de métiers et de fonctions).

2. https://www.lemonde.fr/idees/article/2017/12/26/claude-hagege-ce-n-est-pas-la-langue-quiest-sexiste_5234408_3232.html

3. Notons que nous employons épicène selon la terminologie de Corbett (1991), c'est-à-dire qu'un substantif est seulement compatible avec un genre grammatical (masculin ou féminin) mais peut renvoyer à tout genre social.

4. La liste complète de ces stimuli se trouve en annexe.

5. Un filler - ou une phrase filler - est un item servant simplement de distracteur lors d'une expérience. Ainsi, les participantes et participants ne sont pas uniquement soumis·es aux items testés, ce qui évite qu'elles et ils portent leur attention sur ce qui est véritablement testé.

6. Voir notamment Matell \& Jacoby (1972) pour une discussion sur les différentes échelles.

7. L'erreur standard de la moyenne mesure à quel point la moyenne de l'échantillon des données est éloignée de la véritable moyenne de la population.

8. Dans une autre étude (Burnett et Richy en préparation), nous avons refait cette expérience en alternant genre masculin/féminin, et nous avons trouvé à peu près les mêmes résultats.

\section{RÉSUMÉS}

Cet article présente un nouveau paradigme d'expériences psycholinguistiques qui nous a permis de décortiquer l'effet du genre grammatical et des stéréotypes de genre dans l'interprétation de référents humains en français.

Si les grammaires prescriptives (e.g. Académie Française) et traditionnelles (Grevisse \& Goose 2008) défendent l'idée selon laquelle le masculin est aussi le genre neutre ou « indifférencié » de la langue française, la littérature féministe (e.g. Houdebine-Gravaud 1995) et les récents travaux de psycholinguistique (e.g. Gygax et al. 2008; Gygax et al. 2019) contestent cette position et argumentent que le masculin déclenche en réalité un biais favorisant les hommes dans l'interprétation. Nos expériences vont dans le sens de ces dernières études : le masculin y est aussi interprété comme référant aux hommes. En outre, elles permettent de quantifier pour la première fois pour les contextes où le nom est dépourvu de marque de genre, la contribution du genre grammatical (tant masculin que féminin) aux croyances des locuteurs et locutrices francophones.

This paper presents a new psycholinguistic experimental paradigm, allowing to untangle the impact of grammatical gender and gender stereotypes on human-referring noun phrases in French.

On the one hand, traditional and prescriptive grammars (e.g. Académie Française, Grevisse \& Goose 2008) argue that masculine grammatical gender in French is a neutral or "undifferentiated" gender. On the other hand, feminist literature (e.g. Houdebine-Gravaud 1995) and recent psycholinguistic studies (e.g. Gygax et al. 2008; Gygax et al. 2019) have argued that masculine grammatical gender triggers a mental representation of male referents. Our experiments align with these studies: masculine gender is also interpreted as referring to men. In 
addition, our experimental paradigm is the first to enable to quantify the semantic contribution of grammatical gender (both masculine and feminine) to the meaning of its referent, vis a vis French speakers' belief.

\section{INDEX}

Thèmes : Recherches

Keywords : grammatical gender, French, gender stereotypes, gender bias, psycholinguistics Mots-clés : genre grammatical, français, stéréotypes de genre, biais de genre, psycholinguistique

\section{AUTEURS}

\section{CÉLIA RICHY}

Queen Mary University of London

Célia Richy est doctorante en sociolinguistique à l'Université Queen Mary de Londres et travaille actuellement sur les relations entre langue, lieux et idéologies linguistiques, particulièrement concernant les variétés régionales du Sud-Ouest de la France.

\section{HEATHER BURNETT}

CNRS \& LLF Université de Paris

Heather Burnett est directrice de recherches au CNRS dans le Laboratoire de Linguistique Formelle. Elle travaille sur la modélisation mathématique du social et la construction de l'identité par le langage. 